\title{
Ultra-Low-Leakage and High-Performance Logic Circuit Design Using Multiparameter Asymmetric FinFETs
}

\author{
SOURINDRA M. CHAUDHURI and NIRAJ K. JHA, Princeton University
}

Recently, multigate field-effect transistors have started replacing traditional planar MOSFETs to keep pace with Moore's Law in deep submicron technology. Among different multigate transistors, FinFETs have become the preferred choice of the semiconductor industry owing to low fabrication cost, superior performance, lower leakage, and design flexibility. The back and front gates of a FinFET can either be shorted or remain independent, leading to two modes of operation: Shorted-Gate (SG) and Independent-Gate (IG). For a given mode of operation, the physical parameters of the FinFET can either be symmetric or asymmetric in nature. In this article, for the first time, we analyze multiparameter asymmetric SG FinFETs and illustrate their potential for implementing logic gates and circuits that are both ultra-low-leakage and high-performance simultaneously. We restrict this work to SG devices because IG FinFETs (symmetric/asymmetric) suffer from severely degraded on-current, which makes them unattractive for high-performance designs. We first compare head-to-head all viable single- and multiparameter symmetric/asymmetric SG FinFETs. Among all such FinFETs, the traditional SG (which are symmetric in nature), Asymmetric Workfunction Shorted-Gate (AWSG), and Asymmetric Workfunction-Underlap Shorted-Gate (AWUSG) FinFETs show the most promise. We characterize these devices under process variations in gate length $\left(L_{G}\right)$, fin thickness $\left(T_{S I}\right)$, gate-oxide thickness $\left(T_{O X}\right)$, gate underlap $\left(L_{U N}\right)$, and gate-workfunction $\left(\Phi_{G}\right)$ as well as supply voltage $\left(V_{D D}\right)$ variations, followed by a gate-level leakage/delay analysis at different temperatures. Although AWSG FinFETs consume very low leakage power, they do suffer from performance degradation relative to SG FinFETs. Similarly, our study reveals that no other single-parameter asymmetric FinFET provides a good combination of low-power and high-performance design. We show that gates/circuits based on AWUSG FinFETs are faster, yet consume much less leakage power and less area than gates/circuits based on traditional SG FinFETs. We observe $53.4 \%(30.2 \%)$ maximum (average) reduction in total power at temperature $T=348 \mathrm{~K}$ while meeting the same delay constraint, with $14.2 \%(13.5 \%)$ reduction in area for AWUSG circuits relative to SG circuits. At $T=373 K$, we see $68.6 \%$ (46.9\%) maximum (average) reduction in total power.

Categories and Subject Descriptors: B.6.1 [Design Styles]: Multiparameter Asymmetric FinFET Logic Circuit Design

General Terms: Design, Experimentation, Performance

Additional Key Words and Phrases: FinFETs, multiparameter asymmetry, low-leakage, high-performance, design

ACM Reference Format:

Sourindra M. Chaudhuri and Niraj K. Jha. 2016. Ultra-low-leakage and high-performance logic circuit design using multiparameter asymmetric FinFETs. J. Emerg. Technol. Comput. Syst. 12, 4, Article 43 (March 2016), 25 pages.

DOI: http://dx.doi.org/10.1145/2832913

This work was supported by NSF under Grant No. CCF-1318603.

Authors' addresses: S. M. Chaudhuri and N. K. Jha, Electrical Engineering Department, Princeton University, Princeton, NJ, 08544; emails: \{schaudhu, jha\}@princeton.edu.

Permission to make digital or hard copies of part or all of this work for personal or classroom use is granted without fee provided that copies are not made or distributed for profit or commercial advantage and that copies show this notice on the first page or initial screen of a display along with the full citation. Copyrights for components of this work owned by others than ACM must be honored. Abstracting with credit is permitted. To copy otherwise, to republish, to post on servers, to redistribute to lists, or to use any component of this work in other works requires prior specific permission and/or a fee. Permissions may be requested from Publications Dept., ACM, Inc., 2 Penn Plaza, Suite 701, New York, NY 10121-0701 USA, fax +1 (212) 869-0481, or permissions@acm.org.

(c) 2016 ACM 1550-4832/2016/03-ART43 $\$ 15.00$

DOI: http://dx.doi.org/10.1145/2832913 


\section{INTRODUCTION}

FinFETs have recently been adopted as the most viable among the multigate transistor alternatives [Nowak et al. 2004] by most of the leading semiconductor companies at the $22 \mathrm{~nm}$ technology node and beyond. FinFETs are favored at deep submicron technology nodes primarily because of their tighter control over the channel, thus resulting in low leakage, superior performance, low cost due to seamless transition from the old fabrication process, and design flexibility. FinFETs also offer a plethora of device choices with unique features. This opens up new research opportunities for novel circuit designs.

Both bulk and Silicon-on-Insulator (SOI) FinFETs are being used. In this work, we focus on SOI FinFETs (Figure 1). Such FinFETs have a metal gate that wraps around the thin silicon fin that stands on an insulating base. When a thick spacer material is used at the top, it acts like a double-gate device controlled using both the front and back gates. When the top spacer is not used, the device can be controlled from the top as well and becomes a Trigate device (favored by Intel). Since the FinFET channel is either undoped or lightly doped, the effect of random dopant fluctuations, which is a big concern in bulk CMOS, is mitigated.

In SG FinFETs, the front and back gates are connected at the top (as shown in Figure 1), whereas in IG FinFETs, the top part is etched away, leading to independent front and back gates. SG FinFETs have much higher on-current $\left(I_{O N}\right)$, whereas IG FinFETs have much lower off-current $\left(I_{O F F}\right)$ but very poor $I_{O N}$. The back gate of an IG FinFET can be forward (reverse) biased to dynamically change its $I_{O N} / I_{O F F}$. However, its $I_{O N}$ is still far inferior to the $I_{O N}$ of an SG FinFET.

FinFETs can also be classified as symmetric or asymmetric, independent of their SG or IG modes of operation. Some parameters within the same FinFET that may assume different values are $L_{U N}, \Phi_{G}$, and source/drain doping $\left(N_{S D}\right)$. These devices are called asymmetric. These parameters assume the same value within a symmetric FinFET. We may further classify asymmetric FinFETs as single- or multiparameter asymmetric. In single-parameter asymmetric devices, only one of the parameters takes different values, whereas in multiparameter asymmetric devices, more than one parameter takes nonidentical values. So far, researchers have primarily explored FinFETs with just one of the several parameters being asymmetric, such as AWSG, Asymmetric Doping SG (ADSG), and Asymmetric Underlap SG (AUSG). AWSG FinFETs and logic gates based on them, which are much better placed in the leakage-delay spectrum relative to symmetric SG devices, have shown promise in power/performance optimization of logic circuits, flip-flops, latches, and SRAMs. However, ADSG and AUSG FinFETs have only been explored in the context of SRAM cell design.

FinFETs offer a panoply of choices spanned by SG/IG modes of operation and asymmetric single- and multiparameter devices. They offer very different combinations of $I_{O N} / I_{O F F}$ pairs. However, unless runtime threshold voltage $\left(V_{t h}\right)$ control is desired or the independent front and back gates are fed separate signals to implement an OR operation, any of the preceding combinations involving an IG FinFET would be much inferior to the corresponding combination involving an SG FinFET in terms of $I_{O N}$, and hence circuit performance. Thus, in this article, we only characterize various combinations that involve SG FinFETs. It is very important to the semiconductor industry to find transistors that have both high $I_{O N}$ and very low $I_{O F F}$. We show that some of the preceding combinations offer this possibility.

The key contributions of this article are as follows:

-We characterize all possible symmetric and asymmetric SG FinFETs and compare them head-to-head to establish their relative standing in terms of $I_{O N} / I_{O F F}$. Although some single-parameter asymmetric devices have been explored in the 


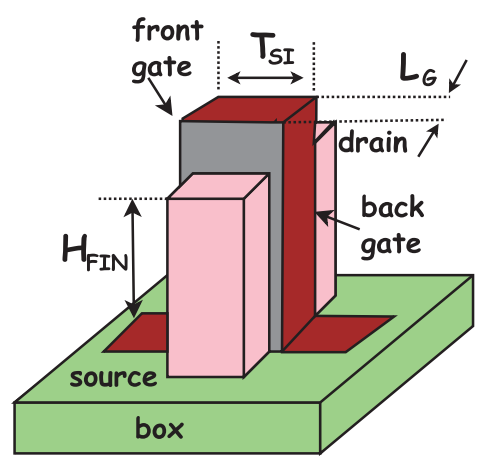

Fig. 1. A typical n/p-FinFET device.

context of SRAMs, for the first time, we introduce the concept and importance of multi-parameter asymmetric FinFETs.

-Traditional SG FinFETs (which are symmetric in nature) are known to provide high $I_{O N}$ but at the price of high $I_{O F F}$. Also, AWSG FinFETs are known to have around $30 \%$ lower $I_{O N}$ but with the advantage of two orders of magnitude lower $I_{O F F}$. Ideally, we would like to work with a transistor that surpasses SG FinFETs in $I_{O N}$ but is competitive with AWSG FinFETs in $I_{O F F}$. We demonstrate that AWUSG is such a FinFET.

-We thoroughly characterize the sensitivity of SG, AWSG, AUSG, and AWUSG FinFETs (among the various FinFET parameter combinations, these FinFETs stood out) to parametric variations in $L_{G}, T_{S I}, T_{O X}, L_{U N}, \Phi_{G}, V_{D D}$, and $T$. Furthermore, we check the robustness of these devices under the effect of process variations in $L_{G}$, $T_{S I}, T_{O X}, L_{U N}$, and $\Phi_{G}$, as well as variations in $V_{D D}$. We then perform leakage-delay analysis of logic gates made of these devices to establish their relative standing in the leakage-delay spectrum at different temperatures.

-Finally, we compare SG and AWUSG FinFET logic circuit implementations to demonstrate significant area/power reductions in the latter at the same delay constraint.

The rest of the article is organized as follows. In Section 2, we review related work. In Section 3, we give a detailed description of different types of FinFETs and characterize various symmetric/asymmetric FinFETs. In Section 4, we analyze logic gates made of single- and novel multiparameter asymmetric FinFETs. In Section 5, we establish the potential of AWUSG devices in optimizing circuit power, area, and performance. Finally, in Section 6, we conclude.

\section{RELATED WORK}

The promise of multigate transistors has been recognized by the research community and semiconductor industry for some time. Introduction of any new device typically demands attention in areas like fabrication, device modeling, simulation techniques, circuit design, and architectural implementation [Bhattacharya and Jha 2014]. Researchers have already fabricated various types of FinFETs, which include devices operated in both SG and IG modes. Although they have primarily focused on symmetric devices so far, the potential of AWSG FinFETs has been recognized through fabrication in the industry [Kedzierski et al. 2001] and characterization by researchers [Bhoj and Jha 2013]. Researchers have also fabricated and demonstrated the superior performance of AUSG MOSFETs [Ghani et al. 2001; Horiuchi et al. 1994].

Appropriate application of device physics and modeling techniques is very critical for FinFETs owing to their nonplanar structure. The 3D nature of FinFETs also makes 
device simulation very CPU intensive. Hence, balancing the accuracy and efficiency of device simulation is a current challenge. To address this problem, in Chaudhuri and Jha [2014a], we proposed an $L_{U N}$ adjustment-based methodology that bridges the accuracy gap between $3 \mathrm{D}$ and $2 \mathrm{D}$ device simulation even under process variations, thus making FinFET device simulations very efficient without much loss in accuracy.

$L_{G}, T_{S I}, T_{O X}$, and $\Phi_{G}$ are considered critical parameters for determining device behavior under process variations [Xiong and Bokor 2003; Mishra et al. 2010]. A response surface-based methodology has been developed to model leakage/delay in FinFET logic gates [Chaudhuri et al. 2012]. The interplay between process-induced and statistical variations has been studied in Wang et al. [2013] and its impact on SRAM stability has been explored further in Wang et al. [2014]. The promise of various FinFET types has motivated researchers to explore their impact at the circuit level to reap powerperformance-stability benefits. Thus, leakage-delay tradeoffs based on the SG/IG modes of operation in FinFET circuits have been explored [Chiang et al. 2005; Kumar et al. 2004; Tawfik and Kursun 2007; Mishra et al. 2009]. In Mishra et al. [2009], Ouyang and Xie [2008], Swahn and Hassoun [2006], and Muttreja et al. [2007], gate sizing and multiple supply/threshold voltage methodologies have been shown to be effective at power optimization. The effect of temperature on device/gate leakage current $\left(I_{L E A K}\right)$ has been explored in Ananthan and Roy [2006], Choi et al. [2007], and Gu et al. [2008]. The concept of multithreshold FinFETs has been explored for obtaining a better leakage/performance profile [Gaynor and Hassoun 2014] under certain restrictions. Recently, orthogonal to the concept of using multithreshold FinFETs (that use different gate workfunctions in different transistors in the IC), AWSG FinFETs (that use two different gate workfunctions in the same FinFET) have been identified as an ultra-low-leakage device. They enable extremely low power designs, but with some loss in performance. The leakage-delay spectrum of logic gates, latches, and flip-flops based on AWSG FinFETs has been investigated in Bhoj and Jha [2013]. It has been shown that AWSG FinFET-based circuits operating at a higher supply voltage compared to SG FinFET-based circuits consume less total power under the same delay constraint [Chaudhuri and Jha 2014b]. In Bhoj and Jha [2014], AWSG-based SRAM bitcells are shown to be competitive in terms of both de metrics and dynamic writeability. ADSG and AUSG FinFETs have been proposed and analyzed in the context of robust SRAM design in Goel et al. [2011] and Moradi et al. [2011]. The impact of Gate-Induced Drain Leakage (GIDL) has been investigated for various symmetric and asymmetric FinFETs in Choi et al. [2003]. The impact is shown to be insignificant in digital circuits operating at $V_{D D}<1.1 \mathrm{~V}$. Electrical characteristics of ADSG FinFETs have been studied in Woo et al. [2002].

In this work, we propose various multiparameter asymmetric FinFETs for the first time. We further compare, analyze, and characterize these novel devices against existing FinFET device options. AWUSG FinFETs emerge from this characterization as the most attractive device from both the $I_{O N}$ and $I_{O F F}$ perspectives. We then show the advantage of AWUSG FinFETs at the logic gate and circuit levels from the area/delay/power perspectives.

\section{SYMMETRIC AND ASYMMETRIC FinFETs}

In this section, we investigate various symmetric and asymmetric FinFETs operating in the SG mode and compare them head-to-head to explore their potential in optimizing both leakage and performance. We use a Python-based integrated simulation platform from Bhoj and Jha [2013] that connects a Sentaurus TCAD Structure Editor (SE), device simulator, SVisual, and MATLAB to ease data flow among these tools [Synopsys 2011]. In our device-level characterization, we simulate a typical SG nFinFET (as shown in Figure 2) through 3D device simulations. For logic gate-level 


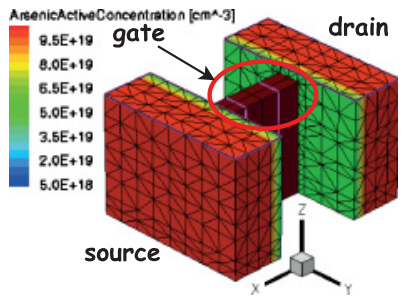

Fig. 2. $3 \mathrm{D}$ nFinFET device created by SE.

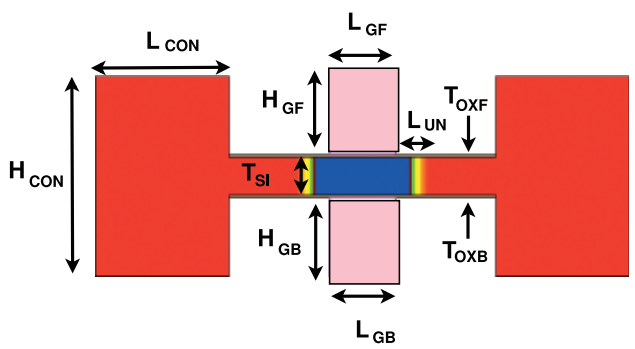

Fig. 3. $2 \mathrm{D}(\mathrm{X}-\mathrm{Y})$ cross-section of the $3 \mathrm{D}$ nFinFET device.

Table I. Typical Symmetric FinFET Device Parameter Values

\begin{tabular}{cc}
\hline \hline Parameter $($ Unit) & Value \\
\hline$L_{G F}, L_{G B}(\mathrm{~nm})$ & 20 \\
$T_{O X F}, T_{O X B}(\mathrm{~nm})$ & 1 \\
$T_{S I}(\mathrm{~nm})$ & 11 \\
$H_{F I N}(\mathrm{~nm})$ & 40 \\
$H_{G F}, H_{G B}(\mathrm{~nm})$ & 24 \\
$L_{U N}(\mathrm{~nm})$ & 10 \\
$F_{P}(\mathrm{~nm})$ & 60 \\
$N_{B O D Y}\left(\mathrm{~cm}^{-3}\right)$ & $10^{15}$ \\
$\Phi_{G}(\mathrm{eV})$ & nFET: $4.4, \mathrm{pFET}: 4.8$ \\
$N_{S D}\left(\mathrm{~cm}^{-3}\right)$ & $10^{20}$ \\
$V_{D D}(\mathrm{~V})$ & 0.9 \\
\hline \hline
\end{tabular}

mixed-mode analysis, we simulate a $2 \mathrm{D}$ cross-section (as shown in Figure 3 ) of the $3 \mathrm{D} \mathrm{n} / \mathrm{p}$-FinFETs for drastically improved simulation efficiency. The analysis is based on hydrodynamic device simulations, which consider the bandgap narrowing model, Philips-unified model for carrier mobility, and Shockley-Read-Hall and band-to-band tunneling models to accurately capture the effect of carrier recombination [Synopsys 2011].

Table I shows typical physical parameter values of a symmetric SG $\mathrm{n} / \mathrm{pFinFET}$ at the $22 \mathrm{~nm}$ technology node, which are calibrated based on data from the foundries [Guillorn et al. 2008; Wu et al. 2010; Yamashita et al. 2011]. In our prior work [Chaudhuri and Jha $2014 \mathrm{a}$, we presented drain-current $I_{D S}$ versus gate voltage $V_{G S}$ curves that are in agreement with the data presented in the literature. Referring to Figure 3, the process parameters are front (back) physical gate length $L_{G F}\left(L_{G B}\right)$, fin thickness $\left(T_{S I}\right)$, front (back) gate oxide thickness $T_{O X F}\left(T_{O X B}\right)$, front (back) gate thickness $H_{G F}\left(H_{G B}\right)$, and $L_{U N}$. $W_{N E F F}$ and $W_{P E F F}$ denote the effective widths of an nFinFET and pFinFET, respectively, and are defined as $2 n H_{F I N}$ (where $n$ is the number of fins in the FinFET and $H_{F I N}$ is the fin height) for an SG FinFET (whether symmetric or asymmetric). These effective widths take into account both the front and back inversion channels 


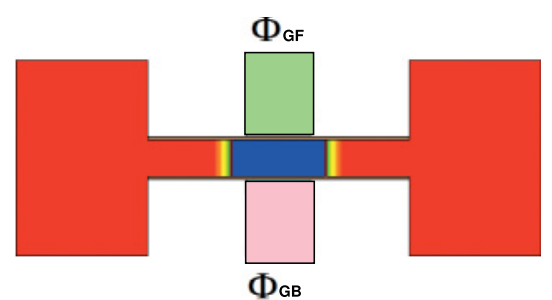

(a)

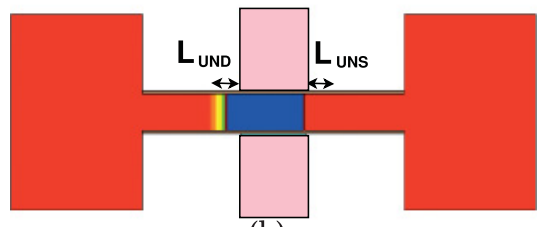

(b)

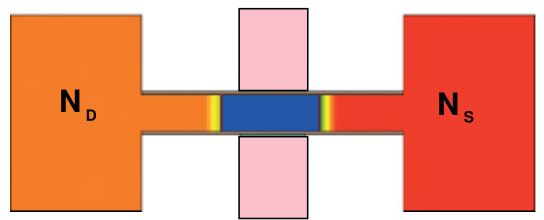

(c)

Fig. 4. 2D cross-section of an (a) AWSG nFinFET, (b) AUSG nFinFET, and (c) ADSG nFinFET.

that are formed in a typical FinFET. Other device parameters are body doping $N_{B O D Y}$, source/drain doping $N_{S D}$, fin pitch $F_{P}$ that denotes the minimum distance required between the midpoint of two adjacent fins, and operating voltage $V_{D D}$.

Among the various physical parameters, $\Phi_{G}, L_{U N}$, and $N_{S D}$ can potentially assume different values within the same FinFET, giving rise to different asymmetric FinFETs. Hence, for asymmetric FinFETs, we refer to these parameters as front/back gate workfunction $\left(\Phi_{G F} / \Phi_{G B}\right)$, source/drain gate underlap $\left(L_{U N S} / L_{U N D}\right)$, and source/drain doping $\left(N_{S} / N_{D}\right)$. FinFETs in which just one physical parameter is asymmetric are classified as single-parameter asymmetric. Examples of such devices are AWSG, AUSG, and ADSG FinFETs, which are shown in Figure 4. On the other hand, FinFETs in which more than one physical parameter are asymmetric are classified as multiparameter asymmetric. Some examples are Asymmetric GateWorkfunction/Gate-Underlap SG (AWUSG), Asymmetric Gate-Workfunction/Doping SG (AWDSG), Asymmetric Gate-Underlap/Doping SG (AUDSG), and Asymmetric Gate-Workfunction/Gate-Underlap/Doping SG (AWUDSG) FinFETs, as shown in Figure 5. It is possible to adjust the workfunction of the gate material, which determines the $V_{t h}$ of the device. For symmetric SG FinFETs (which are the traditional FinFETs), $4.4 \mathrm{eV}(4.8 \mathrm{eV})$ is chosen as the workfunction for nFinFETs (pFinFETs) to implement high-performance logic. In FinFETs that incorporate asymmetric workfunctions, the front and back gates are associated with these different workfunctions, respectively. Table II shows typical values these asymmetric parameters can take, based on the optimizations performed in Bhoj and Jha [2013], Goel et al. [2011], Moradi et al. [2011], Ghani et al. [2001], Horiuchi et al. [1994], and Woo et al. [2002]. However, note that the exact choice of asymmetric parameter values is orthogonal to the study of FinFET asymmetry, which is the focus of this work. As we will see later, the advantages of 


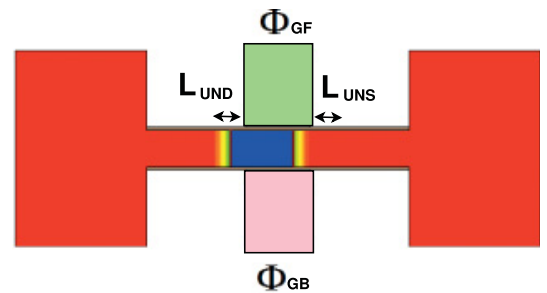

(a)

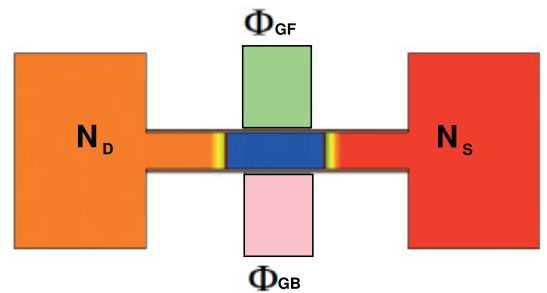

(b)
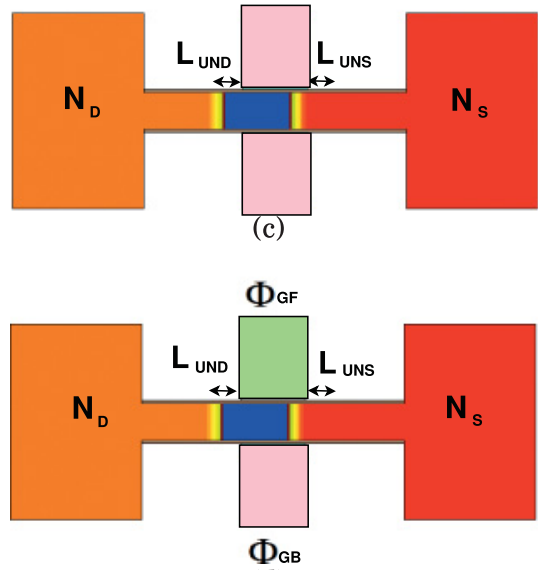

(d)

Fig. 5. 2D cross-section of an (a) AWUSG nFinFET, (b) AWDSG nFinFET, (c) AUDSG nFinFET, and (d) AWUDSG nFinFET.

\begin{tabular}{|c|c|}
\hline Parameter (Unit) & Value \\
\hline$\Phi_{G F}(e V)$ & 4.4 \\
\hline$\Phi_{G B}(e V)$ & 4.8 \\
\hline$L_{U N S}(n m)$ & 0 \\
\hline$L_{U N D}(n m)$ & 10 \\
\hline$N_{S}\left(\mathrm{~cm}^{-3}\right)$ & $10^{20}$ \\
\hline$N_{D}\left(\mathrm{~cm}^{-3}\right)$ & $10^{19}$ \\
\hline
\end{tabular}

asymmetric FinFETs are maintained even when the parameter values are changed within a range.

AWSG FinFETs were originally fabricated and investigated by IBM researchers [Kedzierski et al. 2001]. They fabricated both symmetric and asymmetric devices to 
establish the similarity in their fabrication process. Symmetric device fabrication was carried out on a $65 \mathrm{~nm}$ SOI wafer, topped by a 50nm oxide hard mask. Optical lithography and hard mask trimming were used to define fin layers. A high tilt angle was used to implant extended regions that include multiple wafer twists. On the other hand, asymmetric device fabrication was done on a $120 \mathrm{~nm}$ wafer along with a $90 \mathrm{~nm}$ oxide hard mask. The fins were defined using the same procedure that was used in the case of symmetric devices. Likewise, similar techniques were used to implant extended regions and poly gates. Because of the significant similarities in the fabrication processes of symmetric and asymmetric devices, a seamless transition to asymmetric FinFETs is feasible if desired. Researchers have also fabricated and investigated AUSG planar MOSFETs [Horiuchi et al. 1994; Ghani et al. 2001]. Such asymmetric devices can be fabricated by just following conventional planar CMOS fabrication steps.

\subsection{Single-parameter Asymmetric FinFETs}

In this section, we first analyze the traditional symmetric SG FinFETs in light of device physics to better understand their $I_{O N} / I_{O F F}$ characteristics. We then investigate various single-parameter asymmetric FinFETs-AWSG, AUSG, and ADSG-to look for a FinFET with a better $I_{O N}$ than a symmetric SG FinFET but with a drastically lower $I_{O F F}$. We perform hydrodynamic mixed-mode $3 \mathrm{D}$ device simulations to account for temperature effects using refined meshes optimized for convergence. We ignore gate tunneling effects since the channel is undoped and because gate leakage is negligible due to the use of a relatively thicker oxide made up of high- $\kappa$ dielectric.

Figure 6 shows the electrostatic potential distribution and electron density within the fin region of an SG nFinFET in the OFF-state $\left(V_{D S}=0.9 \mathrm{~V}, V_{G S}=0 \mathrm{~V}\right)$ and in the $\mathrm{ON}$-state $\left(V_{D S}=0.9 \mathrm{~V}, V_{G S}=0.9 \mathrm{~V}\right)$. Figure $6(\mathrm{~b})$ clearly shows severe band bending near both the front and back gates, which helps form prominent inversion regions, as can be seen from Figure 6(d), leading to a high $I_{O N}$. On the contrary, in Figure 6(a), we observe a high potential barrier in the region adjacent to both gates, leaving very little room for a leakage path. However, the potential barrier decreases significantly toward the fin center, enabling a prominent leakage path and giving rise to a high $I_{O F F}$. We obtained an $I_{O N}$ of $146.16 \mu A(76.81 \mu A)$ and $I_{O F F}$ of $1.83 n A(0.11 n A)$ for the SG nFinFET (pFinFET).

In Bhoj and Jha [2013], AWSG FinFETs and logic gates/latches/flip-flops were thoroughly investigated, demonstrating superior subthreshold behavior and ultra-low leakage. Here, we revisit characteristics and related physics of such devices to better understand their limitations in achieving both very low $I_{O F F}$ and high enough $I_{O N}$. AWSG FinFETs are fabricated through an adjustment of gate workfunctions on front and back sides using selective implantation. Thus, they have two different workfunctions on the two sides of the gate [Bhoj and Jha 2013]. The same two workfunctions are used on the two sides of both AWSG nFinFETs and pFinFETs to reduce fabrication cost. Hence, in our experiments, both AWSG n/pFinFETs are assumed to have $4.4 \mathrm{eV}$ and $4.8 \mathrm{eV}$ gate workfunctions, with source/drain doping determining the majority carrier in the ON-state. Use of asymmetric workfunctions leads to a very steep subthreshold slope [Bhoj and Jha 2013]. This results in a very low $I_{O F F}$ but at the price of a slight degradation in $I_{O N}$. Figure 7 shows the electrostatic potential and electron density in the fin region during the OFF- and ON-states of the AWSG FinFET. In Figure 7(b), we observe a similar band bending as in the case of the SG FinFET. This forms the inversion region adjacent to the gate, as can be seen from Figure 7(d) but is somewhat less severe. This results in a degraded $I_{O N}$. On the other hand, AWSG gates display superior OFF-state behavior because no prominent leakage path in the fin region (not even in the fin center) is present, as can be seen from Figure 7(c). We obtain a $28.1 \%(34.7 \%)$ lower $I_{O N}$ of $105.12 \mu \mathrm{A}(50.16 \mu \mathrm{A})$ and $97.8 \%(97.3 \%)$ lower $I_{O F F}$ of $0.04 n A(0.003 n A)$ for 


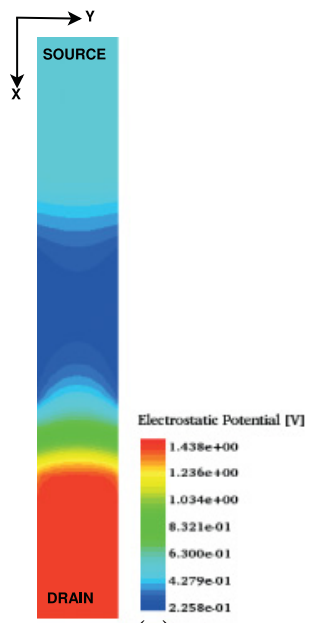

(a)

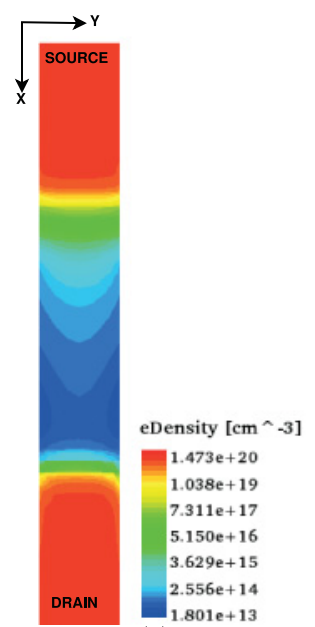

(c)

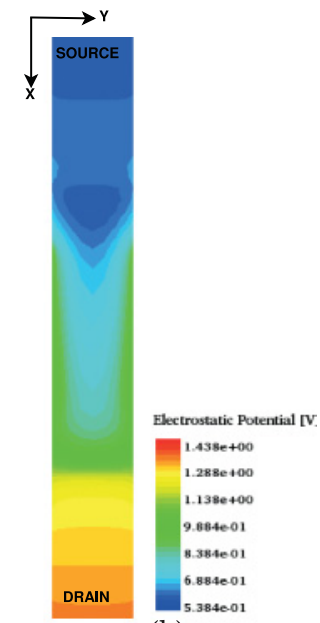

(b)

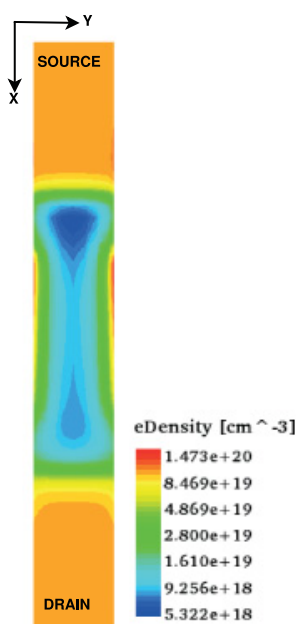

(d)

Fig. 6. Electrostatic potential within the fin region of an SG nFinFET in the (a) OFF-state and (b) ON-state. Electron density within the fin region of an SG nFinFET in the (c) OFF-state and (d) ON-state.

an AWSG nFinFET ( $p$ FinFET) relative to the SG nFinFET (pFinFET). Thus, although AWSG FinFETs are wonderfully suited to ultra-low-leakage design, they are not very well suited to high-performance design due to their degraded $I_{O N}$.

Next, we explore AUSG and ADSG FinFETs, which were proposed in Goel et al. [2011] and Moradi et al. [2011]. AUSG FinFETs have a gate overlap on one side and an underlap on the other side. They can be fabricated using standard FinFET fabrication process steps. However, to introduce an underlap on the drain side, an additional asymmetric spacer formation or tilt ion implantation step is required. An asymmetric spacer can be formed by depositing spacer material on the drain side with a mask on the source side. Further, spacer material can be etched using reactive ion etching [Goel et al. 2011]. Because of this asymmetry, appropriate biasing of these devices is important, yielding different operating configurations for $\mathrm{n} / \mathrm{p}-\mathrm{FinFETs}$. Maintaining $V_{D S}>0\left(V_{D S}<0\right)$ for the nFinFET $($ pFinFET) with an underlap on the drain side yields a higher $I_{O N}$ relative to SG FinFETs, with a minor increase in $I_{O F F}$ [Goel et al. 


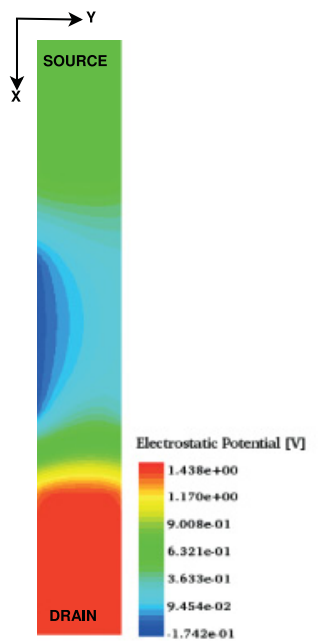

(a)

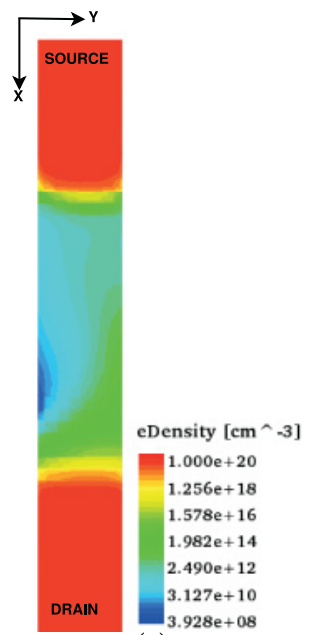

(c)

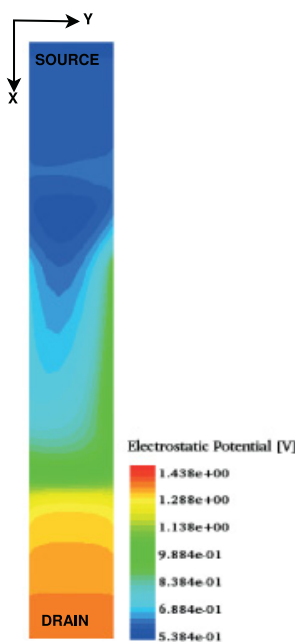

(b)

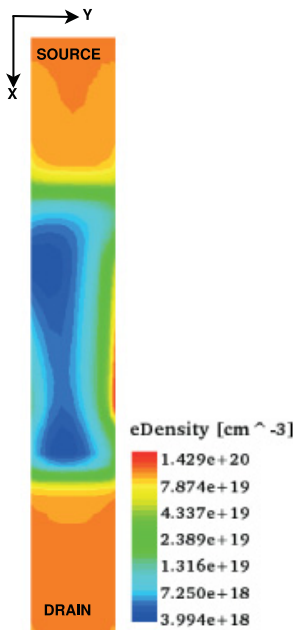

(d)

Fig. 7. Electrostatic potential within the fin region of an AWSG nFinFET in the (a) OFF-state and (b) ONstate. Electron density within the fin region of an AWSG nFinFET in the (c) OFF-state and (d) ON-state.

2011]. An SRAM implemented with such FinFETs at the $7 \mathrm{~nm}$ node has recently been investigated thoroughly [Goud et al. 2015]. Figure 8(b) shows massive band bending, which results in a strong inversion in the fin region, as is evident from Figure 8(d). Figure 8(a) and (c) show the electrostatic potential and electron density in the fin region in the OFF-state. They show a very prominent leakage path in the fin center. Hence, this device is not attractive for low-power design. We obtain a $24.9 \%(49.0 \%)$ higher $I_{O N}$ of $182.55 \mu \mathrm{A}(114.43 \mu \mathrm{A})$ and $122.4 \%$ (127.3\%) higher $I_{O F F}$ of $4.07 n A(0.25 n A)$ for an AUSG nFinFET (pFinFET) compared to an SG nFinFET (pFinFET). This observation shows its potential to be competitive with SG FinFETs if somehow its $I_{O F F}$ could be brought down without compromising much on $I_{O N}$.

In Moradi et al. [2011], an ADSG FinFET was proposed that uses asymmetric doping on the two sides of the device. Table II shows typical doping values used for this type of FinFET. Maintaining $V_{D S}>0\left(V_{D S}<0\right)$ for an nFinFET $(\mathrm{pFinFET})$ with higher doping 


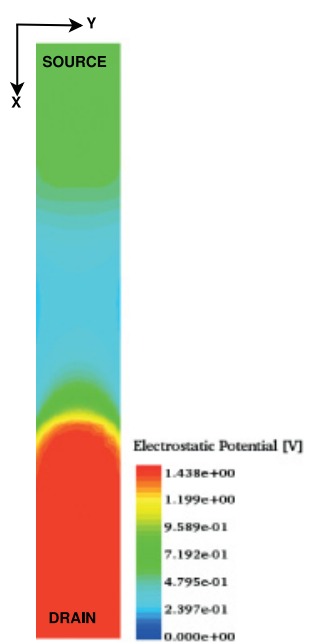

(a)

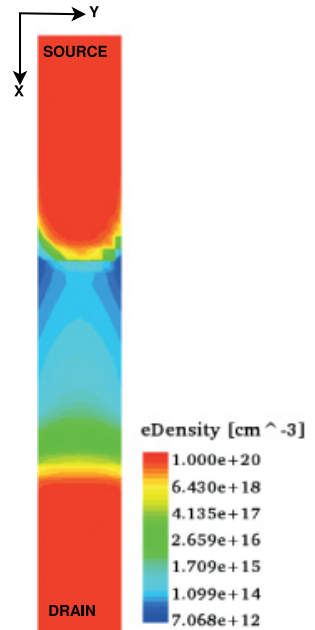

(c)

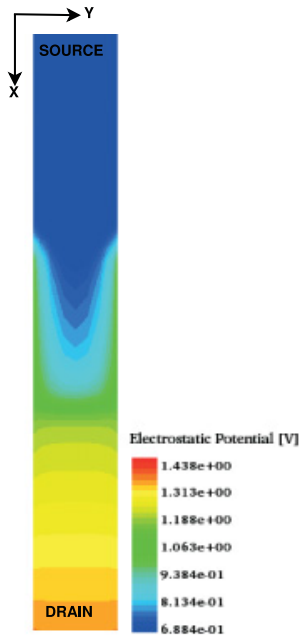

(b)

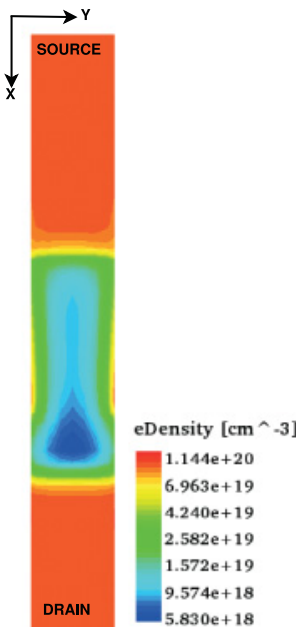

(d)

Fig. 8. Electrostatic potential within the fin region of an AUSG nFinFET in the (a) OFF-state and (b) ONstate. Electron density within the fin region of an AUSG nFinFET in the (c) OFF-state and (d) ON-state.

being on the source side yields better results and is, hence, assumed here. Figure $9(\mathrm{~d})$ shows weaker inversion in the ON-state in the fin region relative to the SG FinFET and, hence, a much lower $I_{O N}$. However, the OFF-state electron density is somewhat identical to that of the SG FinFET. This explains comparable $I_{O F F}$ values. We obtain a $37.1 \%(32.1 \%)$ lower $I_{O N}$ of $91.98 \mu A(52.19 \mu A)$ and $26.8 \%(27.3 \%)$ lower $I_{O F F}$ of $1.34 n A$ $(0.08 n A)$ for an ADSG nFinFET (pFinFET) relative to an SG nFinFET (pFinFET). The significantly lower $I_{O N}$ makes the ADSG FinFET less competitive for high-performance designs. In addition, although ADSG FinFETs are competitive with AWSG FinFETs in $I_{O N}$, they are not at all competitive in $I_{O F F}$.

In Figures 10 and 11, we compare $I_{D S}$ versus $V_{G S}$ characteristics of both n- and ptype single-parameter asymmetric FinFETs and the SG FinFET. Table III summarizes $I_{O F F} / I_{O N}$ results for all these FinFETs (the $1 \times$ suffix denotes the fact that the FinFETs have just one fin). We observe that the AUSG (AWSG) FinFET is superior to an SG 


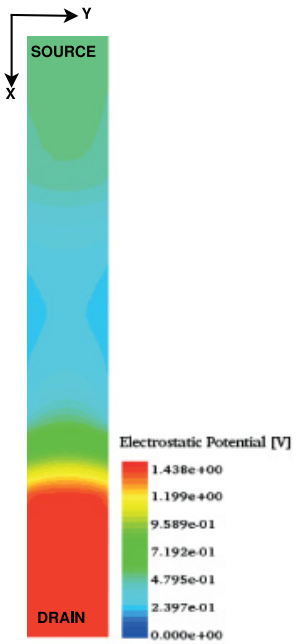

(a)

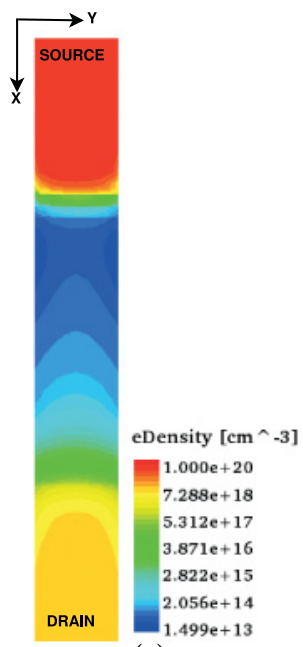

(c)

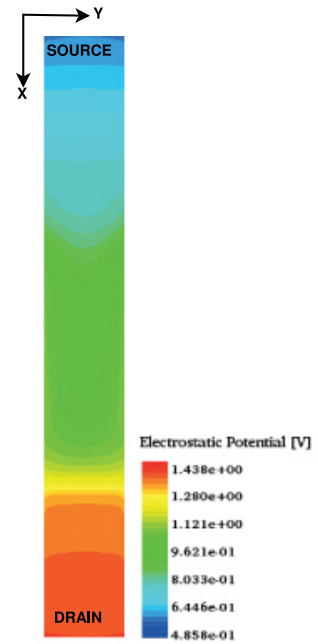

(b)

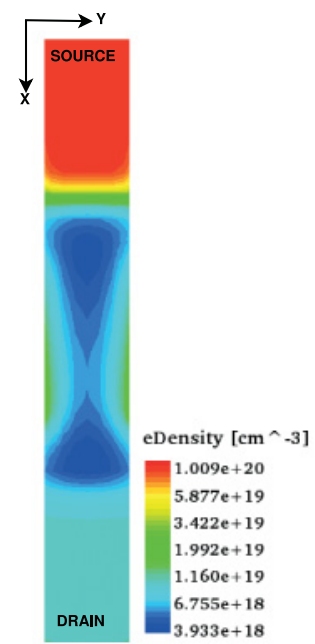

(d)

Fig. 9. Electrostatic potential within the fin region of an ADSG nFinFET in the (a) OFF-state and (b) ONstate. Electron density within the fin region of an ADSG nFinFET in the (c) OFF-state and (d) ON-state.

FinFET in $I_{O N}\left(I_{O F F}\right)$. Thus, if we could combine the $I_{O N}$ advantage of an AUSG FinFET with the $I_{O F F}$ advantage of an AWSG FinFET, we could achieve high performance and low power simultaneously in circuits built with the new FinFET. This motivates us to explore multiparameter asymmetric FinFETs.

\subsection{Multiparameter Asymmetric FinFETs}

So far, we have considered asymmetries in $L_{U N}, \Phi_{G}$, and $N_{S D}$. If we combine such asymmetries in a single FinFET, multiparameter asymmetric FinFETs result (e.g., AWDSG, AWUSG, AUDSG, and AWUDSG). In Table IV, we report $I_{O F F} / I_{O N}$ results of all such n/pFinFETs and compare them to SG FinFETs. In Figures 12 and 13, we compare $I_{D S}$ versus $V_{G S}$ characteristics of all these FinFETs. We observe very low $I_{O F F}$ for AWUSG, AWDSG, and AWUDSG n/pFinFETs. However, only AWUSG FinFETs 


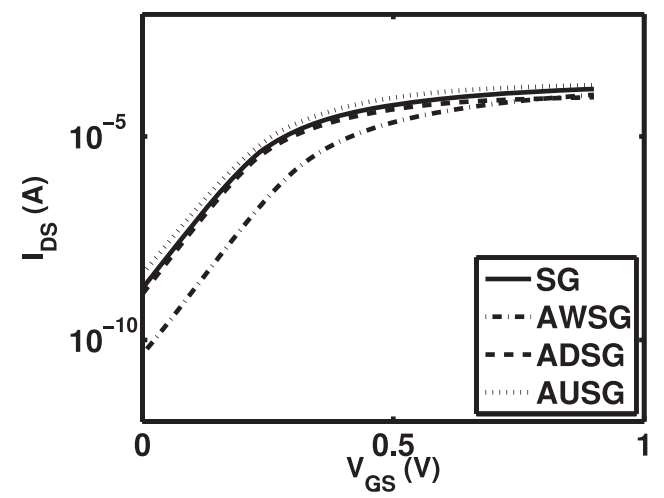

Fig. 10. $I_{D S}$ versus $V_{G S}$ of symmetric and single-parameter asymmetric nFinFETs.

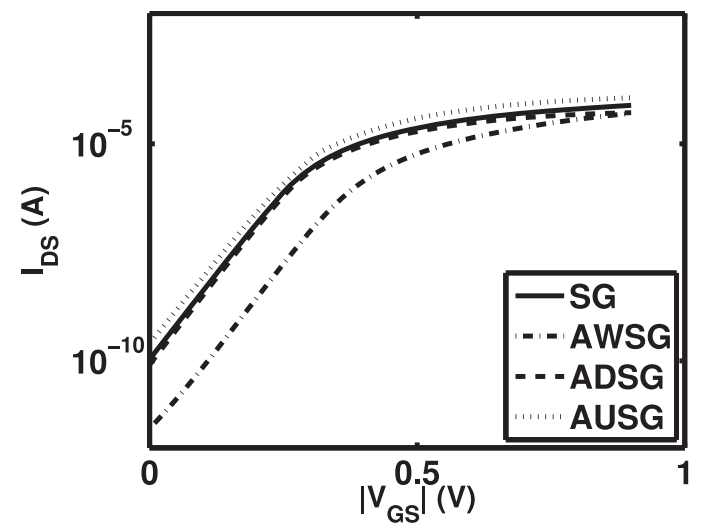

Fig. 11. $I_{D S}$ versus $V_{G S}$ of symmetric and single-parameter asymmetric pFinFETs.

Table III. IOFF/ION of Various Symmetric and Single-Parameter Asymmetric FinFETs

\begin{tabular}{|c|c|c|c|c|c|}
\hline Type & Style & $I_{O F F}(n A)$ & $\%$ Change & $I_{O N}(\mu A)$ & \% Change \\
\hline \multirow{4}{*}{ nFinFET1× } & SG & 1.83 & - & 146.16 & - \\
& AWSG & 0.04 & -97.8 & 105.12 & -28.1 \\
& AUSG & 4.07 & 122.4 & 182.55 & 24.9 \\
& ADSG & 1.34 & -26.8 & 91.98 & -37.1 \\
\hline \multirow{5}{*}{ pFinFET1× } & SG & 0.11 & - & 76.81 & - \\
& AWSG & 0.003 & -97.3 & 50.16 & -34.7 \\
& AUSG & 0.25 & 127.3 & 114.43 & 49.0 \\
& ADSG & 0.08 & -27.3 & 52.19 & -32.1 \\
\hline
\end{tabular}

have competitive $I_{O N}$ with SG FinFETs. The AWUSG nFinFET (pFinFET) has similar (11.6\% higher) $I_{O N}$ to an SG nFinFET (pFinFET), yet a 94.0\% (90.9\%) lower $I_{O F F}$. Thus, this suggests a FinFET that can simultaneously enable high performance and ultralow-leakage power (and, hence, low overall power). Such an observation can also be explained by understanding the qualitative attributes of AWSG and AUSG FinFETs. We discussed earlier how an imbalance in the gate workfunction leads to a very low $I_{O F F}$ in an AWSG FinFET. Similarly, we also saw how an unequal underlap in an AUSG FinFET effectively increases the supply of carriers in the channel region during the 
Table IV. IOFF/ION of Various Multiparameter Asymmetric FinFETs

\begin{tabular}{|c|c|c|c|c|c|}
\hline Type & Style & $I_{O F F}(n A)$ & $\%$ Change & $I_{O N}(\mu A)$ & $\%$ Change \\
\hline \multirow{5}{*}{ nFinFET1× } & SG & 1.83 & - & 146.16 & - \\
& AWDSG & 0.03 & -98.4 & 77.88 & -46.7 \\
& AWUSG & 0.11 & -94.0 & 144.76 & -0.1 \\
& AUDSG & 3.44 & 88.0 & 99.62 & -31.8 \\
& AWUDSG & 0.07 & -96.2 & 89.79 & -38.6 \\
\hline \multirow{5}{*}{ pFinFET1× } & SG & 0.11 & - & 76.81 & - \\
& AWDSG & 0.001 & -99.1 & 40.62 & -47.1 \\
& AWUSG & 0.01 & -90.9 & 85.72 & 11.6 \\
& AUDSG & 0.22 & 100.00 & 60.82 & -20.8 \\
& AWUDSG & 0.004 & -96.4 & 52.20 & -32.0 \\
\hline
\end{tabular}

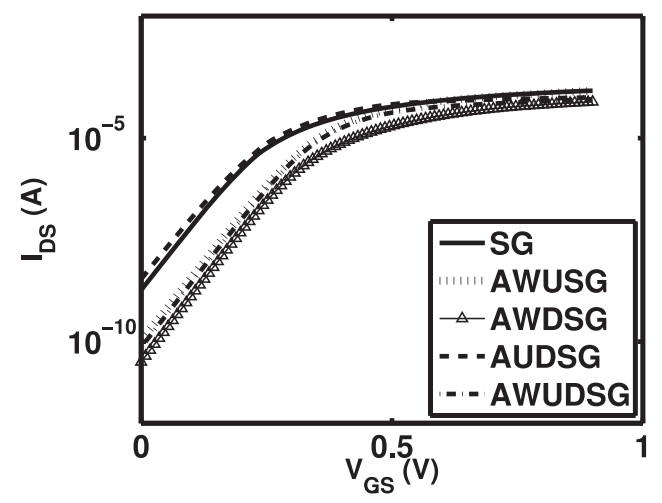

Fig. 12. $I_{D S}$ versus $V_{G S}$ of symmetric and multiparameter asymmetric nFinFETs.

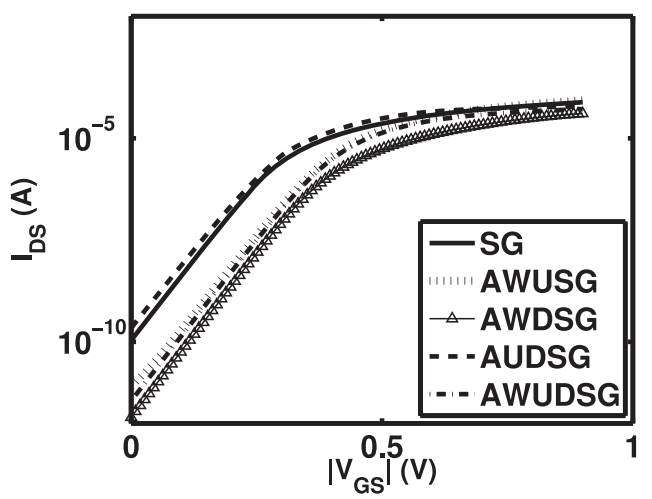

Fig. 13. $I_{D S}$ versus $V_{G S}$ of symmetric and multiparameter asymmetric pFinFETs.

ON-state, which translates into a very high $I_{O N}$. Hence, when we combine these two features in an AWUSG FinFET, we get the best of both worlds.

We show the electrostatic potential and electron density distribution of an AWUSG nFinFET in the fin region in Figure 14. Figure 14(b) shows strong band bending in the ON-state, as in an SG nFinFET, which results in a strong inversion near the gate, as evident from Figure 14(d). This explains why the $I_{O N}$ of such devices is competitive 


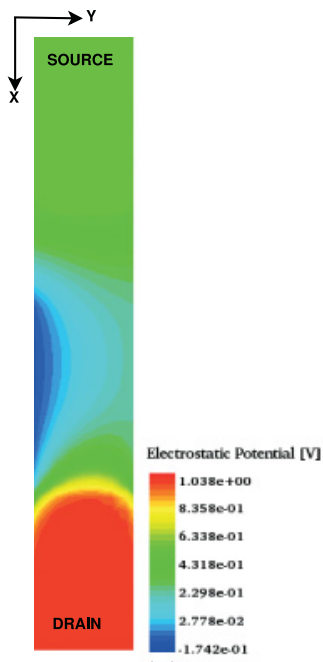

(a)

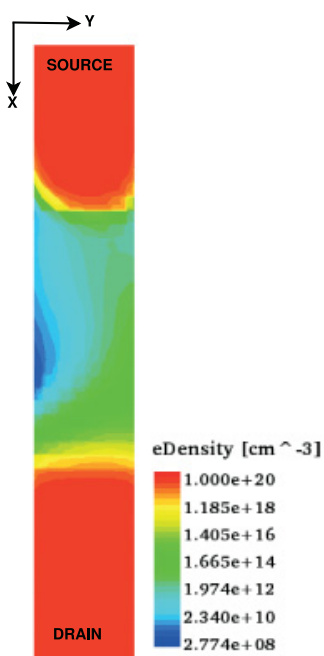

(c)

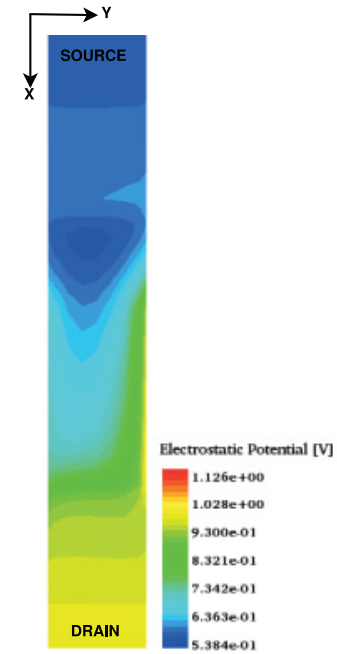

(b)

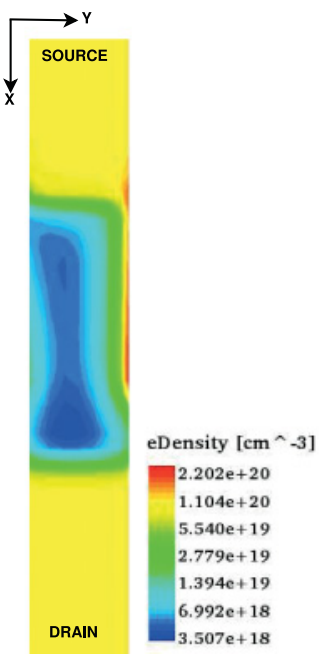

(d)

Fig. 14. Electrostatic potential within the fin region of an AWUSG nFinFET in the (a) OFF-state and (b) ON-state. Electron density within the fin region of an AWUSG nFinFET in the (c) OFF-state and (d) ON-state.

with that of SG FinFETs. In addition, Figure 14(c) shows no prominent leakage path in the OFF-state. It possesses an electron density distribution similar to that of the AWSG FinFET. Thus, the AWUSG FinFET combines the benefits of a higher $I_{O N}$ of the SG FinFET with a lower $I_{O F F}$ of the AWSG FinFET.

In Table $\mathrm{V}$, we report $V_{t h}$ and subthreshold slope $(S S)$ of all single-/multiparameter asymmetric FinFETs along with those of the SG FinFET. All FinFETs have very good subthreshold characteristics. However, n/p-type AWSG and AWUSG FinFETs have relatively high $V_{t h}$ values, which explains their very low $I_{O F F}$ values. We obtain the $S S$ for these devices through regression analysis. We probe the characteristics curve every $10 \mathrm{mV}$ in the subthreshold region and then obtain a linear fit. The slope of the linear fit gives us the $S S$ of the device. 
Table V. $V_{\text {th }} /$ SS of Various Asymmetric n/pFinFETs

\begin{tabular}{|c|c|c|c|c|}
\hline Style & $V_{t h n}(V)$ & $S S_{n}(m V /$ dec $)$ & $V_{t h p}(V)$ & $S S_{p}(m V /$ dec $)$ \\
\hline SG & 0.27 & 68 & -0.34 & 69 \\
AWSG & 0.42 & 69 & -0.52 & 70 \\
AUSG & 0.27 & 71 & -0.34 & 72 \\
ADSG & 0.27 & 68 & -0.32 & 66 \\
AWUSG & 0.34 & 66 & -0.42 & 68 \\
AWDSG & 0.39 & 63 & -0.42 & 64 \\
AUDSG & 0.26 & 70 & -0.31 & 68 \\
AWUDSG & 0.33 & 61 & -0.40 & 63 \\
\hline
\end{tabular}

\subsection{Sensitivity to Various Parameter Variations}

In the previous section, we identified a few winning devices: AWSG (best $I_{O F F}$ ), AUSG (best $I_{O N}$ ), and AWUSG (best of both worlds). In this section, we investigate the $I_{O F F} / I_{O N}$ sensitivity of these nFinFETs with respect to variations in various parameters: $L_{G}, T_{S I}, T_{O X}, L_{U N}, \Phi_{G}$, and $V_{D D}$ (pFinFETs exhibit similar sensitivities). We include SG nFinFETs for the sake of comparisons. Figure 15 shows the sensitivity of $I_{O F F}$ to variations in $L_{G}, T_{S I}, T_{O X}, L_{U N}, \Phi_{G}$, and $V_{D D}$. We observe that $I_{O F F}$ decreases significantly with increasing $L_{G}, L_{U N}$, and $\Phi_{G}$; increases significantly with increasing $T_{S I}$; and is relatively indifferent to variations in $T_{O X}$ and $V_{D D}$. It is also evident that $I_{O F F}$ is most sensitive to variations in $\Phi_{G}$ and $T_{S I}$. In Figures $15(\mathrm{e})$, the $x$-axis represents different sets of $\Phi_{G}$ values depending on transistor type. For an SG FinFET, values 1 through 5 on the $x$-axis denote $4.38 \mathrm{eV}, 4.39 \mathrm{eV}, 4.40 \mathrm{eV}, 4.41 \mathrm{eV}$, and $4.42 \mathrm{eV}$, respectively, whereas for other asymmetric FinFETs, they represent a pair of $\Phi_{G}$ values: $4.38 / 4.78 \mathrm{eV}, 4.39 / 4.79 \mathrm{eV}, 4.40 / 4.80 \mathrm{eV}, 4.41 / 4.81 \mathrm{eV}$, and $4.42 / 4.82 \mathrm{eV}$, respectively. We assume that both front- and back-gate workfunctions vary in the same direction based on the assumption that they are realized using a similar process recipe.

In Figure 16, we show the sensitivity of $I_{O N}$ with respect to changes in the various parameters. We see that $I_{O N}$ decreases linearly with increasing $L_{G}$ and $L_{U N}$, but increases roughly linearly with $T_{S I}$ and $V_{D D}$. $I_{O N}$ is not very sensitive to variations in $T_{O X}$ and $\Phi_{G}$.

We also observe that, in the case of $I_{O F F}$ variations, SG and AUSG cluster together, as do AWSG and AWUSG. However, in the case of $I_{O N}$ variations, SG and AWUSG tend to be closer. This is very desirable since it shows that AWUSG FinFETs share the $I_{O F F}$ characteristics of AWSG FinFETs and the $I_{O N}$ characteristics of SG FinFETs under a wide range of parameter values.

\subsection{Sensitivity to Temperature Variation}

$I_{O F F}$ of FinFETs increases exponentially with an increase in temperature $T$. Figure 17 shows this trend. However, AWSG and AWUSG FinFETs maintain their relative advantage over SG and AUSG FinFETs even at higher temperatures. $I_{O N}$ does not change much with $T$. For example, within a wide variation range in $T$ (300-373K), $I_{O N}$ only varies within $10 \%$ for all device types considered here.

\subsection{Effect of Process Variations}

$L_{G}, T_{S I}, T_{O X}, L_{U N}, \Phi_{G}$, and $V_{D D}$ have been shown to be the most susceptible to process variations [Xiong and Bokor 2003; Mishra et al. 2010]. In this section, we analyze the robustness of SG/AWSG/AUSG/AWUSG nFinFET1 $\times$ under combined variations in these parameters. We use Sobol sequence-based quasi-Monte Carlo (QMC) simulation [Singhee and Rutenbar 2007], which is known to need several orders of magnitude fewer samples than Monte Carlo simulation to achieve a similar accuracy, for this 

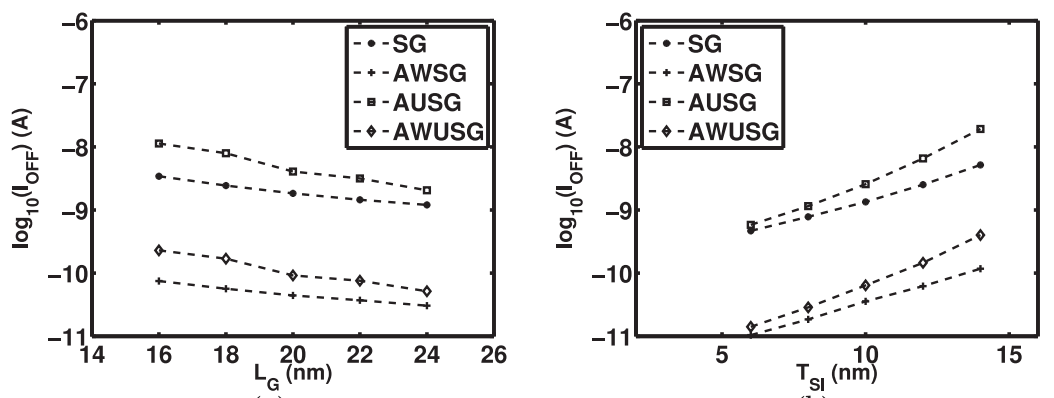

(a)

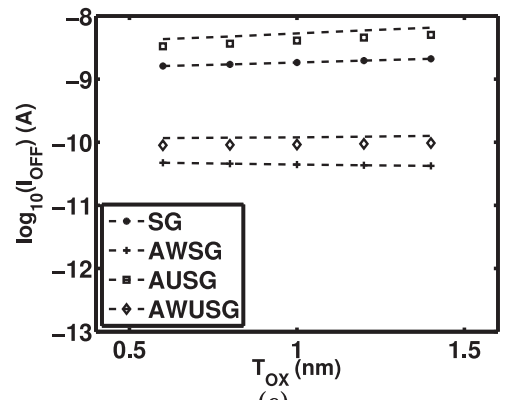

(b)

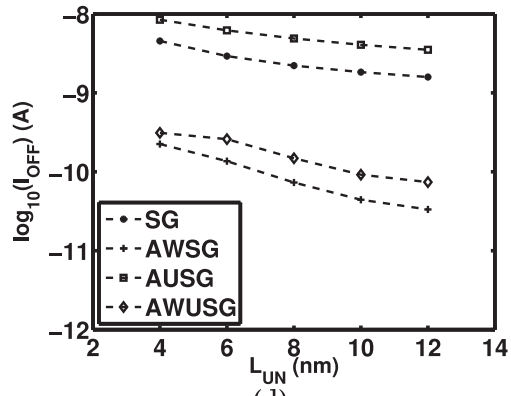

(d)

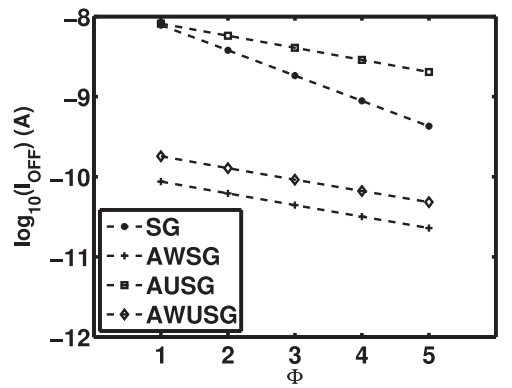

(e)

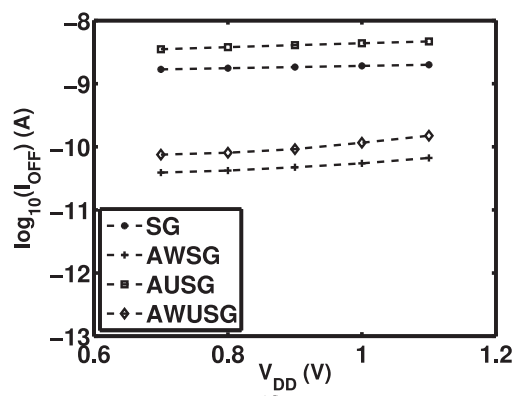

(f)

Fig. 15. Sensitivity of $I_{O F F}$ to variations in (a) $L_{G}$, (b) $T_{S I}$, (c) $T_{O X}$, (d) $L_{U N}$, (e) $\Phi_{G}$, and (f) $V_{D D}$.

analysis. Thus, we use QMC simulation to generate 100 sample variation cases with each parameter having a $3 * \sigma / \mu=10 \%$ variation, and we perform $3 \mathrm{D}$ hydrodynamic device simulations with these values. We show $I_{O F F}$ and $I_{O N}$ distributions under such variations in Figure 18. We can see that the AWUSG FinFET has reasonable $I_{O N} / I_{O F F}$ spreads relative to the other FinFETs.

\section{FinFET LOGIC GATES}

In this section, we study leakage-delay characteristics of logic gates built atop the FinFETs characterized in the previous section. We focus on size $1 / 2 \times$ logic gates. Tables VI and VII show average leakage current (across all input vectors), $I_{L E A K}$, for the $1 \times$ and $2 \times$ logic gates at $300 \mathrm{~K}$ temperature. As expected, AWSG and AWUSG FinFET-based $1 / 2 \times$ logic gates maintain their advantage in this respect, even across temperatures, as seen from Tables VIII, IX, X, and XI. In Tables XII and XIII, we compare the delays of the $1 / 2 \times$ logic gates. We observe a delay reduction of $15.0 \%$ (14.5\%), $4.2 \%(4.8 \%)$, and $12.4 \%(12.5 \%)$ for the AWUSG inverter $1 \times(2 \times)$, NAND $1 \times(2 \times)$, and NOR $1 \times(2 \times)$ 


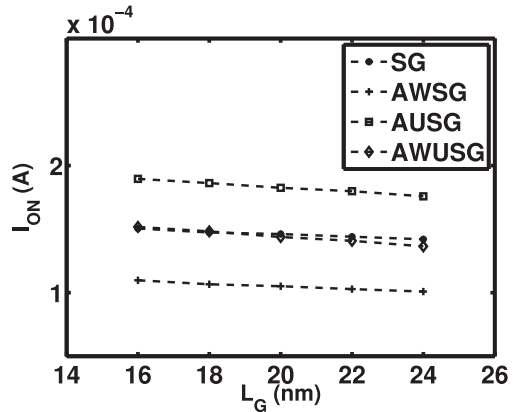

(a)

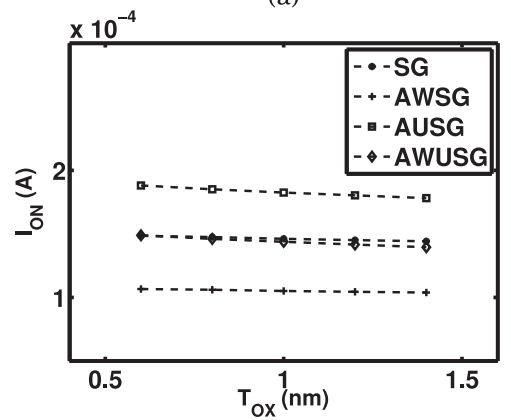

(c)

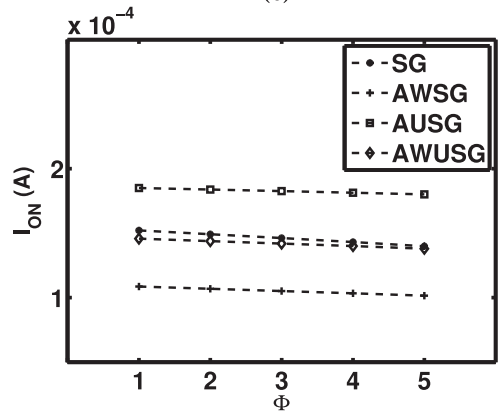

(e)

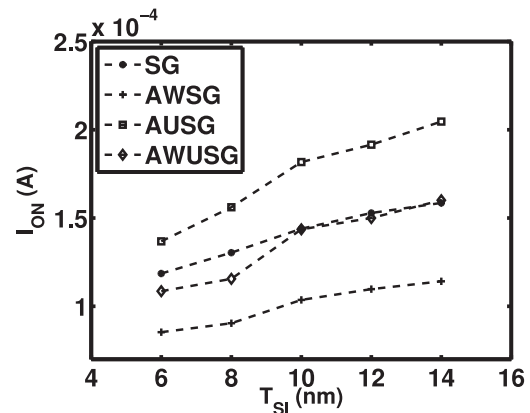

(b)

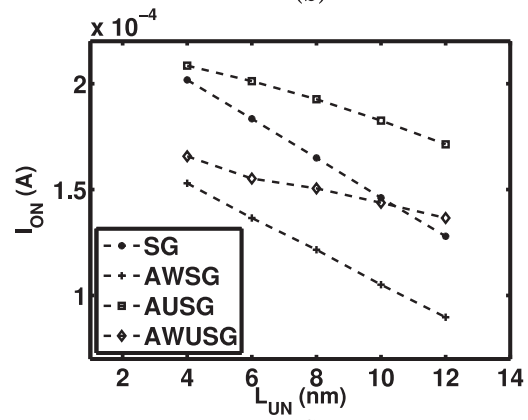

(d)

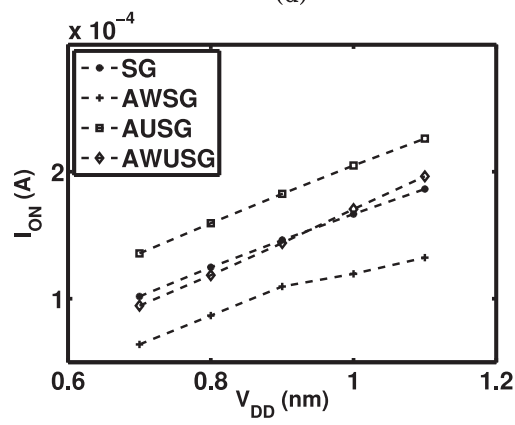

(f)

Fig. 16. Sensitivity of $I_{O N}$ to variations in (a) $L_{G}$, (b) $T_{S I}$, (c) $T_{O X}$, (d) $L_{U N}$, (e) $\Phi_{G}$, and (f) $V_{D D}$.

gates, respectively, relative to the corresponding SG FinFET-based logic gates. Thus, AWUSG FinFETs enable both lower delay and a drastic reduction in leakage current. The $1 \times(2 \times)$ inverter, NAND, and NOR gates, respectively, have $1(2), 1(2)$, and 1 (2) fins in all nFinFETs and 2 (4), 1 (2), and 4 (8) fins in all pFinFETs. These configurations were chosen to account for the difference in hole and electron mobility and yield comparable rise/fall delays. This explains why the NAND gate delay is higher than the NOR gate delay. Note that a $2 \times$ NAND gate has only 8 fins (as opposed to 10 fins in a $1 \times$ NOR gate). Thus, a $2 \times$ NAND gate both occupies less area and is faster than a $1 \times$ NOR gate.

\section{FinFET LOGIC CIRCUITS}

In this section, we evaluate the circuit-level impact of AWUSG FinFETs relative to SG FinFETs. Since AWUSG FinFET-based logic gates are faster, we first obtain the fastest possible circuit implementations based on $1 \times$ logic gates (inverter/NAND/NOR) 


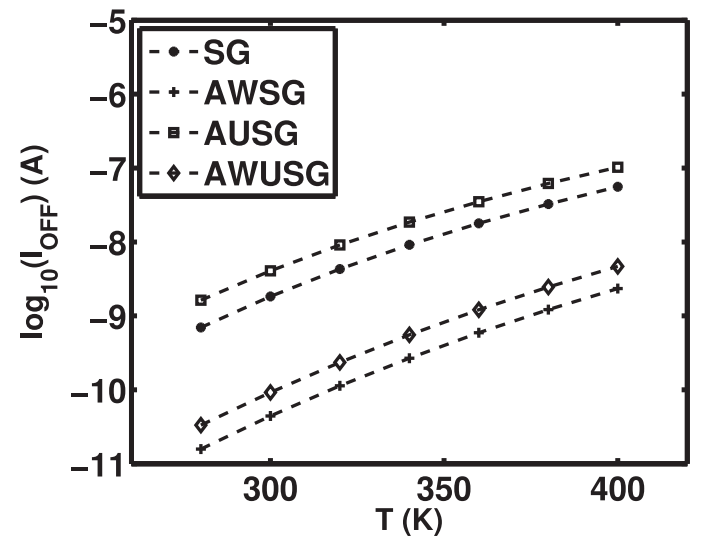

Fig. 17. $I_{O F F}$ versus $T$ of the SG/AWSG/AUSG/AWUSG nFinFET.

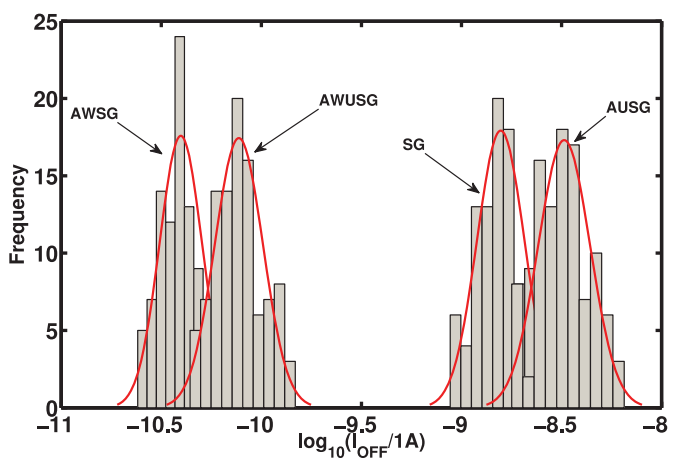

(a)

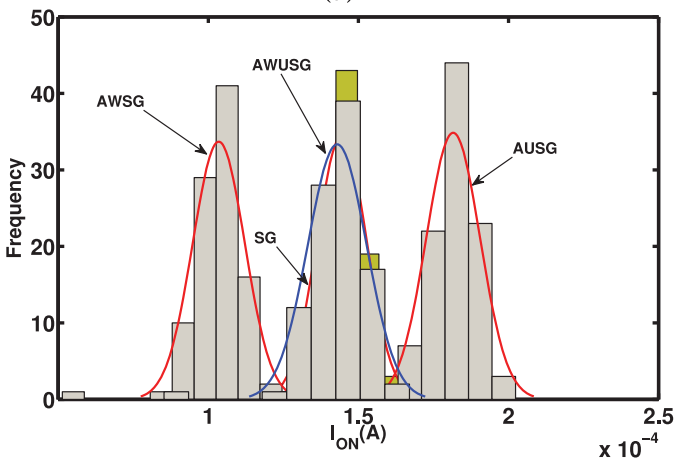

(b)

Fig. 18. (a) $I_{O F F}$ distributions of the SG/AWSG/AUSG/AWUSG nFinFET under process variations, (b) $I_{O N}$ distributions of the SG/AWSG/AUSG/AWUSG nFinFET under process variations.

Table VI. I LEAK Comparisons of $1 \times$ Logic Gates at $T=300 \mathrm{~K}$

\begin{tabular}{|c|c|c|c|c|c|c|}
\hline Style & Inverter $(n A)$ & \% Change & NAND $(n A)$ & \% Change & NOR $(n A)$ & \% Change \\
\hline SG & 1.03 & - & 1.15 & - & 1.18 & - \\
\hline AWSG & 0.02 & -98.1 & 0.03 & -97.4 & 0.03 & -97.5 \\
\hline AUSG & 2.84 & 175.7 & 3.10 & 169.6 & 2.53 & 114.4 \\
\hline AWUSG & 0.06 & -94.2 & 0.06 & -94.8 & 0.06 & -94.9 \\
\hline
\end{tabular}


Table VII. $I_{\text {LEAK }}$ Comparisons of $2 \times$ Logic Gates at $T=300 \mathrm{~K}$

\begin{tabular}{|c|c|c|c|c|c|c|}
\hline Style & Inverter $(n A)$ & \% Change & NAND $(n A)$ & \% Change & NOR $(n A)$ & $\%$ Change \\
\hline SG & 2.05 & - & 2.30 & - & 2.35 & - \\
\hline AWSG & 0.04 & -98.1 & 0.06 & -97.4 & 0.06 & -97.5 \\
\hline AUSG & 5.67 & 176.6 & 6.20 & 169.6 & 5.06 & 115.3 \\
\hline AWUSG & 0.12 & -94.2 & 0.12 & -94.8 & 0.12 & -94.9 \\
\hline
\end{tabular}

Table VIII. I LEAK Comparisons of $1 \times$ Logic Gates at $T=348 \mathrm{~K}$

\begin{tabular}{|c|c|c|c|c|c|c|}
\hline Style & Inverter $(n A)$ & \% Change & NAND $(n A)$ & \% Change & NOR $(n A)$ & \% Change \\
\hline SG & 7.02 & - & 7.75 & - & 8.40 & - \\
\hline AWSG & 0.21 & -97.0 & 0.23 & -97.0 & 0.28 & -96.7 \\
\hline AUSG & 16.51 & 135.2 & 17.93 & 131.4 & 17.93 & 113.5 \\
\hline AWUSG & 0.51 & -92.7 & 0.56 & -92.8 & 0.62 & -92.6 \\
\hline
\end{tabular}

Table IX. I IEAK Comparisons of $2 \times$ Logic Gates at $T=348 \mathrm{~K}$

\begin{tabular}{|c|c|c|c|c|c|c|}
\hline Style & Inverter $(n A)$ & \% Change & NAND $(n A)$ & \% Change & NOR $(n A)$ & \% Change \\
\hline SG & 14.04 & - & 15.49 & - & 16.80 & - \\
\hline AWSG & 0.42 & -97.0 & 0.46 & -97.0 & 0.56 & -96.7 \\
\hline AUSG & 33.03 & 135.3 & 35.85 & 131.4 & 35.85 & 113.4 \\
\hline AWUSG & 1.01 & -92.8 & 1.12 & -92.8 & 1.24 & -92.6 \\
\hline
\end{tabular}

Table X. ILEAK Comparisons of $1 \times$ Logic Gates at $T=373 \mathrm{~K}$

\begin{tabular}{|c|c|c|c|c|c|c|}
\hline Style & Inverter $(n A)$ & \% Change & NAND $(n A)$ & \% Change & NOR $(n A)$ & \% Change \\
\hline SG & 15.81 & - & 17.29 & - & 19.32 & - \\
\hline AWSG & 0.56 & -96.5 & 0.61 & -96.5 & 0.74 & -96.2 \\
\hline AUSG & 34.69 & 119.4 & 37.50 & 116.9 & 38.29 & 98.2 \\
\hline AWUSG & 1.31 & -91.7 & 1.42 & -91.8 & 1.58 & -91.8 \\
\hline
\end{tabular}

Table XI. ILEAK Comparisons of $2 \times$ Logic Gates at $T=373 \mathrm{~K}$

\begin{tabular}{|c|c|c|c|c|c|c|}
\hline Style & Inverter $(n A)$ & \% Change & NAND $(n A)$ & \% Change & NOR $(n A)$ & \% Change \\
\hline SG & 31.62 & - & 34.57 & - & 38.64 & - \\
\hline AWSG & 1.12 & -96.5 & 1.22 & -96.5 & 1.47 & -96.2 \\
\hline AUSG & 69.37 & 119.4 & 75.00 & 117.0 & 76.58 & 98.2 \\
\hline AWUSG & 2.62 & -91.7 & 2.83 & -91.8 & 3.15 & -91.8 \\
\hline
\end{tabular}

Table XII. Delay $\left(t_{D}\right)$ Comparisons of $1 \times$ Logic Gates

\begin{tabular}{|c|c|c|c|c|c|c|}
\hline Style & Inverter $(\mathrm{ps})$ & \% Change & NAND $(\mathrm{ps})$ & \% Change & NOR $(\mathrm{ps})$ & \% Change \\
\hline SG & 5.54 & - & 9.57 & - & 6.36 & - \\
\hline AWSG & 7.09 & 28.0 & 12.88 & 34.6 & 8.27 & 30.0 \\
\hline AUSG & 3.71 & -33.0 & 7.22 & -24.6 & 4.63 & -27.2 \\
\hline AWUSG & 4.71 & -15.0 & 9.17 & -4.2 & 5.57 & -12.4 \\
\hline
\end{tabular}

Table XIII. Delay $\left(t_{D}\right)$ Comparisons of $2 \times$ Logic Gates

\begin{tabular}{|c|c|c|c|c|c|c|}
\hline Style & Inverter $(\mathrm{ps})$ & \% Change & NAND $(\mathrm{ps})$ & \% Change & NOR $(\mathrm{ps})$ & \% Change \\
\hline SG & 2.76 & - & 4.79 & - & 3.19 & - \\
\hline AWSG & 3.53 & 27.9 & 6.43 & 34.2 & 4.14 & 29.8 \\
\hline AUSG & 1.86 & -32.6 & 3.60 & -24.8 & 2.30 & -27.9 \\
\hline AWUSG & 2.36 & -14.5 & 4.56 & -4.8 & 2.79 & -12.5 \\
\hline
\end{tabular}


Table XIV. Critical Path Delay for the Fastest Implementation of ISCAS'85 Benchmarks with AWUSG FinFETs

\begin{tabular}{|c|c|}
\hline Benchmarks & $\begin{array}{c}\text { Critical Path Delay } \\
(\mathrm{ps})\end{array}$ \\
\hline c432 & 660 \\
c499 & 773 \\
c880 & 608 \\
c1355 & 764 \\
c2670 & 648 \\
c3540 & 1161 \\
c5315 & 782 \\
c7552 & 809 \\
\hline
\end{tabular}

Table XV. Critical Path Delay for the Fastest Implementation of ISCAS'89 Benchmarks with AWUSG FinFETs

\begin{tabular}{|c|c|}
\hline Benchmarks & $\begin{array}{c}\text { Critical Path Delay } \\
(\mathrm{ps})\end{array}$ \\
\hline s510 & 116 \\
s641 & 284 \\
s713 & 300 \\
s820 & 134 \\
s832 & 163 \\
s1238 & 480 \\
s1423 & 315 \\
s1488 & 213 \\
s5378 & 444 \\
s15850 & 980 \\
s35932 & 222 \\
s38584 & 869 \\
\hline
\end{tabular}

built using AWUSG FinFETs (these implementations thus also consume least area). Tables XIV and XV show the critical path delay for implementations of ISCAS'85 and ISCAS'89 benchmark circuits, respectively. Next, we set these critical path delays as timing constraints for synthesis using Synopsys Design Compiler [Synopsys 2011] based on an SG library consisting of $1 \times$ and $2 \times$ SG FinFET-based logic gates (inverter/NAND/NOR). Note that since SG FinFET-based logic gates are slower, just using $1 \times$ logic gates would not be enough to meet the timing constraint.

Table XVI shows results for ISCAS'85 benchmarks. $P_{D}$ denotes dynamic power. $P_{L 348}$, $P_{T 348}, P_{L 373}$, and $P_{T 373}$ denote leakage power at $348 \mathrm{~K}$, total power at $348 \mathrm{~K}$, leakage power at $373 K$, and total power at $373 K$, respectively. Although SG implementations meet the timing constraint in all the cases, AWUSG implementations consume $12.0 \%$ less area, on average. More importantly, AWUSG implementations also consume $25.6 \%$ $(41.8 \%)$ lower total power at $348 K(373 K)$, on average. We report results for ISCAS'89 benchmarks in Table XVII. AWUSG implementations consume 14.5\% less area while also consuming $38.2 \%(50.4 \%)$ lower total power at $348 K(373 K)$, on average. Since delay is not that sensitive to temperature in FinFET circuits, we ignore this effect. In any case, since an increasing temperature would lead to a similar minor increase in delay for both SG and AWUSG circuits, it is not important to take this phenomenon into account for a comparative analysis.

We can deploy such libraries to higher level architectural simulation tools like FinCANON [Lee and Jha 2014] and McPAT-PVT [Tang et al. 2015] for a complete 


\begin{tabular}{|c|c|c|c|c|c|c|c|c|}
\hline 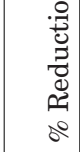 & $\stackrel{9}{\rightarrow}$ & 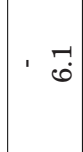 & , $\stackrel{\infty}{\underset{\sim}{+}}$ & $\mid \begin{array}{c}+1 \\
1 \\
1 \\
\end{array}$ & $\begin{array}{ll}0 \\
1\end{array}$ & , & 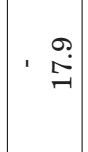 & $\stackrel{\circ}{\stackrel{\circ}{ }}$ \\
\hline 总 & 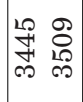 & $\begin{array}{ll}0 & \infty \\
& \infty \\
\infty & \infty \\
\infty & \infty\end{array}$ & 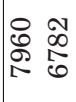 & $\mid \begin{array}{ll}0 & 0 \\
\infty & 1 \\
\infty & 0 \\
\infty & \infty\end{array}$ & 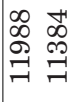 & 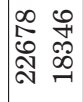 & 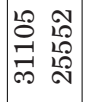 & 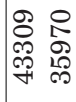 \\
\hline$\therefore$ & 至 8 & $\begin{array}{ll}\infty & \infty \\
\dot{\infty} & \stackrel{2}{1}\end{array}$ & $\begin{array}{ll}1 & \infty \\
\stackrel{0}{0} & \infty \\
0 & 8 \\
0 & 0\end{array}$ & 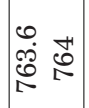 & $\begin{array}{ll}\infty & \\
0 & \infty \\
\text { tै } & +1 \\
0 & 0\end{array}$ & $\begin{array}{ll}0 & -1 \\
\overline{0} & 0 \\
\ddots & -1\end{array}$ & $\mid$\begin{tabular}{cc}
$\infty$ & \\
$\dot{-1}$ & $\infty$ \\
\hdashline & $\infty$ \\
$\Sigma$ & $\infty$
\end{tabular} & $\begin{array}{ll}\infty & \\
\infty & 8 \\
\infty & \infty \\
\infty & \infty\end{array}$ \\
\hline 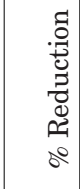 & 点 & , & , $\stackrel{0}{\text { i }}$ & 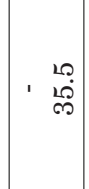 & , $\stackrel{\infty}{\underset{\gamma}{+}}$ & , $\stackrel{\infty}{\mathscr{\gamma}}$ & $\begin{array}{l}0 \\
\dot{\varphi} \\
\dot{H}\end{array}$ & 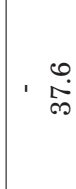 \\
\hline$\frac{\sqrt{2}}{3}$ & 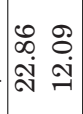 & 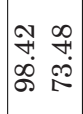 & 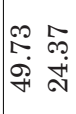 & 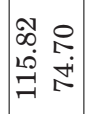 & 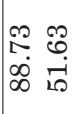 & 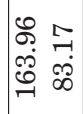 & 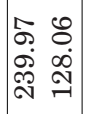 & 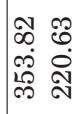 \\
\hline 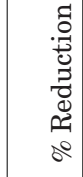 & , & . & $\begin{array}{l}10 \\
\text { i } \\
\text { î }\end{array}$ & , $\stackrel{\overbrace{}}{\stackrel{T}{+}}$ & , $\stackrel{+}{\stackrel{H}{N}}$ & , & , & $\stackrel{\infty}{\stackrel{\sim}{\sim}}$ \\
\hline 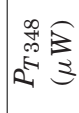 & $\mid \begin{array}{ll}\infty & \Re \\
0 & 0 \\
0 & 0 \\
-1 & -7\end{array}$ & 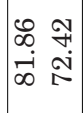 & 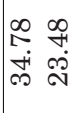 & $\mid$\begin{tabular}{cc}
5 & \multicolumn{1}{c}{} \\
0 & 0 \\
5 & 0 \\
$\sigma$ & 0
\end{tabular} & 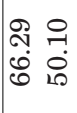 & 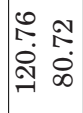 & 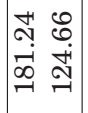 & 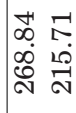 \\
\hline 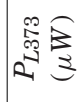 & $\mid \begin{array}{ll}\stackrel{N}{1} & 0 \\
\infty & \infty \\
\text { N丶 } & 0 \\
& 0\end{array}$ & 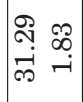 & 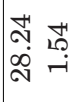 & 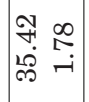 & 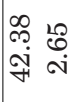 & 岕 & \begin{tabular}{ll}
$\mathscr{8}$ & 0 \\
0 & $\infty$ \\
\hdashline & $\infty$ \\
\hdashline & \llcorner
\end{tabular} & $\begin{array}{ll}10 & 0 \\
0 & 0 \\
0 & 0 \\
0 & \infty\end{array}$ \\
\hline 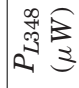 & 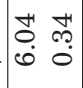 & 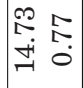 & 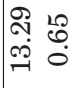 & $\mid \begin{array}{ll}0 & 0 \\
0 & 2 \\
0 & 0 \\
-1 & 0\end{array}$ & 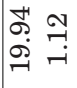 & 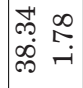 & 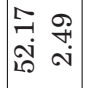 & 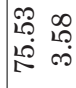 \\
\hline $20 \frac{3}{3}$ & $\mid$\begin{tabular}{ll} 
ㄴ \\
: \\
\hdashline \\
\hdashline
\end{tabular} & 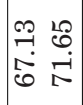 & 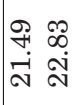 & 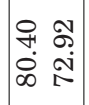 & 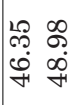 & 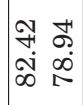 & 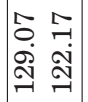 & 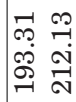 \\
\hline$\therefore \varepsilon$ & 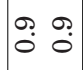 & $\stackrel{0}{9} \stackrel{0}{0}$ & $\stackrel{0}{0} \stackrel{0}{0}$ & $\begin{array}{ll}0 & 0 \\
0 & 0\end{array}$ & $\begin{array}{ll}0 \\
0 \\
0 & 0\end{array}$ & 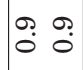 & $\begin{array}{ll}0 & 0 \\
0 & 0\end{array}$ & 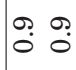 \\
\hline 疋 & 贶 & 资 & 焉 & 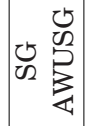 & 党 & ע & 垎 & ש \\
\hline 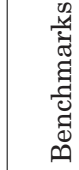 & 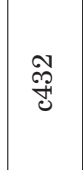 & ஓ্ণ্ডে & $\begin{array}{l}\infty \\
\infty \\
\infty \\
0 \\
0\end{array}$ & 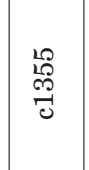 & 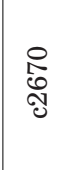 & $\begin{array}{l}\text { 유 } \\
\text { مొ }\end{array}$ & $\begin{array}{l}10 \\
0 \\
0 \\
10 \\
0\end{array}$ & $\begin{array}{l}\text { No } \\
\text { i⿱ } \\
\text { है } \\
\end{array}$ \\
\hline
\end{tabular}




\begin{tabular}{|c|c|c|c|c|c|c|c|c|c|c|c|c|}
\hline 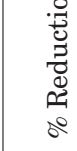 & 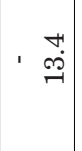 & , $\underset{\sim}{\stackrel{N}{+}}$ & 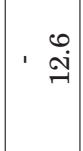 & $\stackrel{H}{\stackrel{H}{S}}$ & 足 & $\mid \begin{array}{ll}\infty \\
1 \\
1 \\
0\end{array}$ & 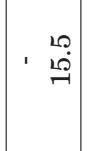 & 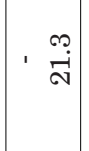 & 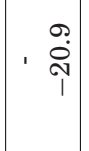 & $\mid \begin{array}{ll}10 \\
1 \\
1 \\
\end{array}$ & 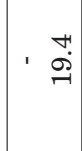 & $\begin{array}{l}\infty \\
\infty \\
\infty \\
\sim\end{array}$ \\
\hline 茪 & 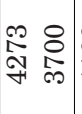 & 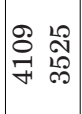 & 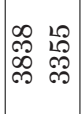 & 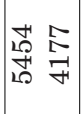 & 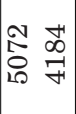 & $\left|\begin{array}{ll}1 & 0 \\
0 & 0 \\
1 & 1 \\
0 & 5 \\
-1 & 0\end{array}\right|$ & $\left|\begin{array}{ll} & 0 \\
\infty & \infty \\
\hdashline & 0 \\
0 & \infty \\
-1 & \infty\end{array}\right|$ & 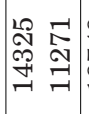 & 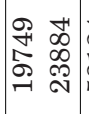 & 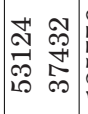 & 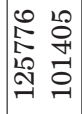 & 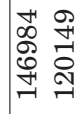 \\
\hline 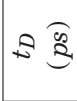 & {$\left[\begin{array}{ll}10 & 0 \\
10 & 0 \\
\exists & \ddots\end{array}\right]$} & $\begin{array}{ll}0 & +1 \\
\infty & \infty \\
\infty & \infty \\
\text { N } & \stackrel{\infty}{N}\end{array}$ & 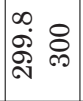 & 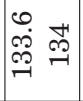 & 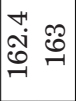 & 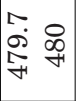 & $\left|\begin{array}{ll}\infty & 20 \\
\dot{H} & 12 \\
\infty & \infty\end{array}\right|$ & 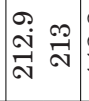 & 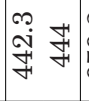 & 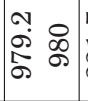 & 곯 న & $\begin{array}{ll}-1 & 0 \\
10 & 8 \\
\infty & 0\end{array}$ \\
\hline 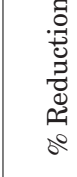 & , $\begin{array}{r}\stackrel{H}{\rightarrow} \\
\end{array}$ & $\begin{array}{l}0 \\
1 \\
0 \\
0\end{array}$ & , 1.7 & $\begin{array}{l}0 \\
\dot{\varphi} \\
\dot{q}\end{array}$ & $\begin{array}{l}10 \\
\text { î } \\
\text { î }\end{array}$ & 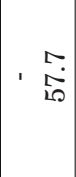 & $\mid \begin{array}{cc}0 \\
1 \\
1 \\
\infty \\
0\end{array}$ & $\left|\begin{array}{cc}1 & -1 \\
1 & \infty \\
\stackrel{N}{0}\end{array}\right|$ & $\begin{array}{ll}1 & 0 \\
1 & \dot{8} \\
10\end{array}$ & $\begin{array}{l}+1 \\
\dot{H}\end{array}$ & 囟 & $\begin{array}{l}\stackrel{0}{\dot{f}} \\
\stackrel{+}{*}\end{array}$ \\
\hline$\frac{2}{0}$ & 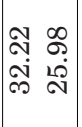 & 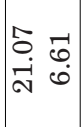 & 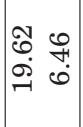 & 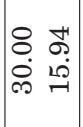 & 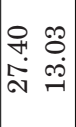 & 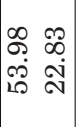 & 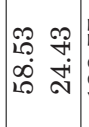 & 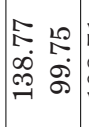 & $\begin{array}{cc} & \infty \\
\infty & 1 \\
0 & 1 \\
0 & \stackrel{\forall}{-1}\end{array} \mid$ & 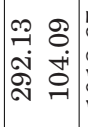 & 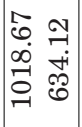 & 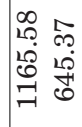 \\
\hline 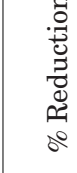 & , $\stackrel{5}{\stackrel{\tau}{i}}$ & 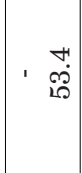 & 1 & 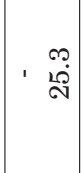 & , & $\mid \begin{array}{cc}\infty \\
1 \\
1 \\
\infty \\
\infty\end{array}$ & $\mid \begin{array}{c}0 \\
1 \\
\\
\dot{\forall}\end{array}$ & 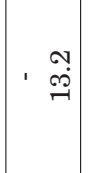 & 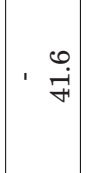 & $\overrightarrow{-1}$ & $\begin{array}{l}\sim \\
\stackrel{\sim}{\mathrm{N}} \\
\stackrel{\mathrm{N}}{2}\end{array}$ & ஓి \\
\hline 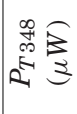 & 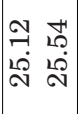 & $\begin{array}{ll}\stackrel{2}{N} & \sim \\
& -1 \\
\rightarrow & 0\end{array}$ & 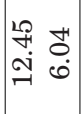 & 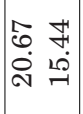 & 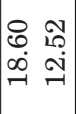 & 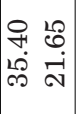 & 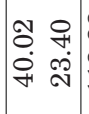 & 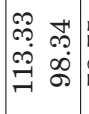 & 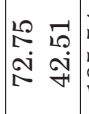 & 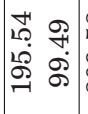 & 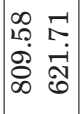 & 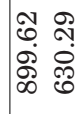 \\
\hline $2^{2} \frac{\sqrt{3}}{3}$ & 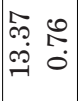 & \begin{tabular}{ll}
$\infty$ & 0 \\
2 & 2 \\
\multirow{H}{*}{} & 0 \\
- & 0
\end{tabular} & 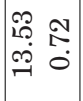 & $\mid \begin{array}{ll}\infty & 0 \\
10 \\
\stackrel{1}{0} & 0 \\
& 0 \\
-1 & 0\end{array}$ & $\mid \begin{array}{ll}\Omega & \infty \\
10 & \infty \\
0 & 0 \\
-1 & 0\end{array}$ & 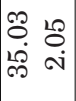 & 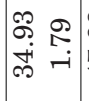 & 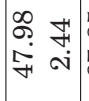 & 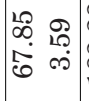 & 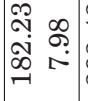 & 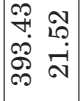 & 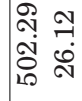 \\
\hline 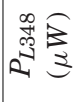 & 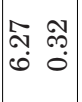 & $\begin{array}{ll}0 & 1 \\
0 & 0 \\
0 & 0\end{array}$ & $\begin{array}{cc}0 & 0 \\
0 & 0 \\
0 & 0 \\
0 & 0\end{array}$ & 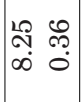 & 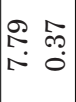 & 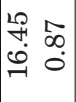 & $\left|\begin{array}{ll}\multirow{1}{*}{} & 0 \\
4 & 0 \\
0 & 2 \\
-1 & 0\end{array}\right|$ & 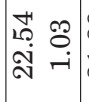 & 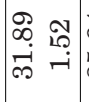 & 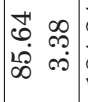 & 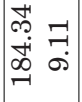 & 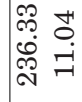 \\
\hline $20 \frac{\sqrt{3}}{3}$ & 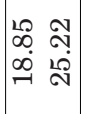 & 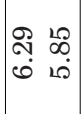 & $\mid \begin{array}{ll}8 & +1 \\
0 & 5 \\
0 & 10 \\
0\end{array}$ & 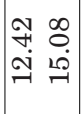 & 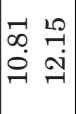 & 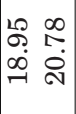 & 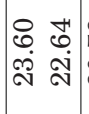 & 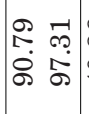 & 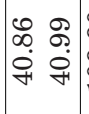 & $\left.\mid \begin{array}{ll}8 & 7 \\
8 & 7 \\
0 & 0 \\
0 & 0\end{array}\right]$ & 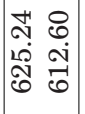 & 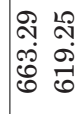 \\
\hline$\therefore \varepsilon$ & $\begin{array}{ll}0 \\
\dot{0} & 0 \\
0\end{array}$ & $\begin{array}{ll}0 \\
\dot{0} & 0 \\
0 & 0\end{array}$ & $\begin{array}{ll}0 \\
\ddot{0} & 0 \\
0 & 0\end{array}$ & \begin{tabular}{ll}
0 \\
\hdashline & 0 \\
0 & 0
\end{tabular} & $\begin{array}{ll}0 & 9 \\
0 & 0\end{array}$ & $\begin{array}{ll}0 & : \\
0 & 0\end{array}$ & $\begin{array}{ll}0 & 9 \\
0 & 0\end{array}$ & $\begin{array}{ll}0 & 9 \\
0 & 0\end{array}$ & $\left.\begin{array}{ll}0 \\
\hdashline\end{array}\right)$ & \begin{tabular}{cc}
0 \\
\hdashline \\
0
\end{tabular} & $\mid \begin{array}{ll}0 & ? \\
0 & 0\end{array}$ & {$\left[\begin{array}{ll}0 & 0 \\
0 & 0\end{array}\right.$} \\
\hline 宓 & 每 & 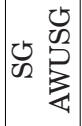 & 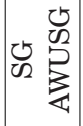 & 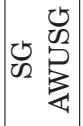 & 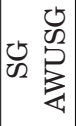 & 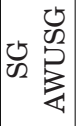 & 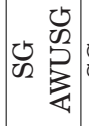 & 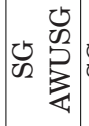 & 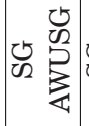 & $\begin{array}{lll} & 5 \\
0 & 0 \\
0 & 3 \\
W & 3\end{array}$ & 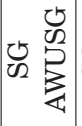 & 先 \\
\hline 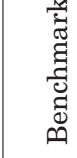 & 量 & 吢 & $\underset{\sigma_{2}}{\infty}$ & 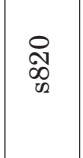 & 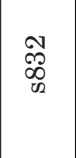 & 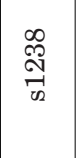 & 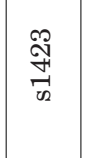 & $\begin{array}{l}\infty \\
\stackrel{\infty}{+} \\
\underset{v n}{4}\end{array}$ & 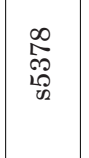 & $\begin{array}{l}0 \\
10 \\
10 \\
10 \\
\rightarrow 1\end{array}$ & 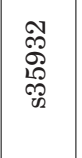 & $\begin{array}{l}\not \\
\infty \\
1 \\
\infty \\
\infty \\
\infty \\
02\end{array}$ \\
\hline
\end{tabular}


Process-Voltage-Temperature (PVT) analysis. FinCANON is a comprehensive tool for the power/delay simulation of caches and on-chip networks under PVT variations. Likewise, McPAT-PVT is an integrated framework for power/delay simulations of FinFET processors under PVT variations.

\section{CONCLUSION}

Although single-parameter asymmetric FinFETs were previously explored in the context of SRAMs, only the AWSG FinFETs were explored in the context of logic circuits. Also, the universe of multiparameter asymmetric FinFETs had not been explored. In this article, for the first time, we performed a thorough analysis of various multiparameter asymmetric FinFETs and discovered AWUSG to be the most valuable for optimizing both performance and leakage. We found that AWUSG FinFETs offer both higher $I_{O N}$ and drastically lower $I_{O F F}$ compared to traditional SG FinFETs. Looking for such transistors has been an important goal of researchers and the semiconductor industry. We analyzed AWUSG FinFETs under process variations and showed they have $I_{O N}$ and $I_{O F F}$ spreads similar to other FinFETs. We then analyzed logic gates based on various promising FinFETs and again found AWUSG logic gates to have the best combined leakage-delay characteristics. We showed that AWUSG circuit implementations consume both less area and significantly less total power relative to SG circuit implementations. The power advantage grows at higher temperatures.

\section{REFERENCES}

H. Ananthan and K. Roy. 2006. A fully physical model for leakage distribution under process variations in nanoscale double-gate CMOS. In Proceedings of the Design Automation Conference. 413-419.

D. Bhattacharya and N. K. Jha. 2014. FinFETs: From devices to architectures. Advances in Electronics (Sep. 2014).

A. N. Bhoj and N. K. Jha. 2013. Design of logic gates and flip-flops in high-performance FinFET technology. IEEE Transactions on VLSI Systems 21, 11 (Nov. 2013), 1975-1988.

A. N. Bhoj and N. K. Jha. 2014. Parasitics-aware design of symmetric and asymmetric gate-workfunction FinFET SRAMs. IEEE Transactions on VLSI Systems 22, 3 (Nov. 2014), 548-561.

S. Chaudhuri and N. K. Jha. 2014a. 3D vs. 2D device simulation of FinFET logic gates under PVT variations. ACM Journal of Emerging Technologies in Computing Systems 10, 3 (Apr. 2014).

S. Chaudhuri and N. K. Jha. 2014b. FinFET logic circuit optimization with different FinFET styles: Lower power possible at higher supply voltage. In Proceedings of the International Conference on VLSI Design. 476-482.

S. Chaudhuri, P. Mishra, and N. K. Jha. 2012. Accurate leakage estimation for FinFET standard cells using the response surface methodology. In Proceedings of the International Conference on VLSI Design. 238-244.

M. H. Chiang, K. Kim, C. Tretz, and C. T. Chuang. 2005. Novel high-density low-power logic circuit techniques using DG devices. IEEE Electronic Device Letter 52, 10 (Oct. 2005), 2339-2342.

J. H. Choi, J. Murthy, and K. Roy. 2007. The effect of process variation on device temperatures in FinFET circuits. In Proceedings of the International Conference on Computer-Aided Design. 747-751.

Y. Choi, D. Ha, T. King, and J. Bokor. 2003. Investigation of gate-induced drain leakage (GIDL) current in thin body devices: Single-gate ultra-thin body, symmetrical double-gate, and asymmetrical double-gate MOSFETs. Japan Journal of Applied Physics 42, 4B (Apr. 2003), 2073-2076.

B. D. Gaynor and S. Hassoun. 2014. Fin shape impact on FinFET leakage with application to multithreshold and ultralow-leakage FinFET design. IEEE Transactions on Electronic Devices 61, 8 (Aug. 2014), 27382744.

T. Ghani, K. Mistry, P. Packan, M. Armstrong, S. Thompson, S. Tyagi, and M. Bohr. 2001. Asymmetric source/drain extension transistor structure for high performance sub-50 nm gate length CMOS devices. VLSI Technology (Jun. 2001), 17-18.

A. Goel, S. Gupta, and K. Roy. 2011. Asymmetric drain spacer extension (ADSE) FinFETs for low-power and robust SRAMs. IEEE Transactions on Electronic Devices 58, 2 (Feb. 2011), 296-308.

A. Goud, R. Venkatesan, A. Raghunathan, and K. Roy. 2015. Asymmetric underlapped FinFET based robust SRAM design at 7nm node. In Proceedings of the Design, Automation Test in Europe Conference. 659-664. 
J. Gu, J. Keane, S. Sapatnekar, and C. H. Kim. 2008. Statistical leakage estimation of double gate FinFET devices considering the width quantization property. IEEE Transactions on VLSI Systems 16 (Feb. 2008), 206-209.

M. Guillorn et al. 2008. FinFET performance advantage at 22nm: An AC perspective. In Proceedings of the Symposium on VLSI Technology. 12-13.

T. Horiuchi, T. Homma, Y. Murao, and K. Okumura. 1994. An asymmetric sidewall process for high performance LDD MOSFET's. IEEE Transactions on Electronic Devices 41, 2 (Feb. 1994), 186-190.

J. Kedzierski et al. 2001. High-performance symmetric-gate and CMOS compatible Vt asymmetric-gate FinFET devices. In Proceedings of the International Electronic Device Meeting 19.5.1-19.5.4.

A. Kumar, B. A. Minch, and S. Tiwari. 2004. Low voltage and performance tunable CMOS circuit design using independently driven double gate MOSFETs. In Proceedings of the International SOI Conference. 119-121.

C.-Y. Lee and N. K. Jha. 2014. FinCANON: A PVT-aware integrated delay and power modeling framework for FinFET-based caches and on-chip networks. IEEE Transactions on VLSI Systems 22, 5 (May 2014), 1150-1163.

P. Mishra, A. N. Bhoj, and N. K. Jha. 2010. Die-level leakage power analysis of FinFET circuits considering process variations. In Proceedings of the IEEE International Symposium on Quality Electronic Design. 347-355.

P. Mishra, A. Muttreja, and N. K. Jha. 2009. Low-power FinFET circuit synthesis using multiple supply and threshold voltages. ACM Journal of Emerging Technologies in Computing Systems 5, 2 (Jul. 2009), 1-23.

F. Moradi, S. Gupta, G. Panagopoulos, D. Wisland, H. Mahmoodi, and K. Roy. 2011. Asymmetrically doped FinFETs for low-power robust SRAMs. IEEE Transactions on Electronic Devices 58, 12 (Dec. 2011), 4241-4249.

A. Muttreja, N. Agarwal, and N. K. Jha. 2007. CMOS logic design with independent-gate FinFETs. In Proceedings of the IEEE International Conference on Computer Design. 560-567.

E. J. Nowak, I. Aller, T. Ludwig, K. Kim, R. V. Joshi, C. T. Chuang, K. Bernstein, and R. Puri. 2004. Turning silicon on its edge. IEEE Circuits and Devices Magazine 20, 1 (Jan. 2004), 20-31.

J. Ouyang and Y. Xie. 2008. Power optimization for FinFET based circuits using genetic algorithms. In Proceedings of the IEEE International SOC Conference. 211-214.

A. Singhee and R. A. Rutenbar. 2007. From finance to flip flops: A study of fast quasi-Monte Carlo methods from computational finance applied to statistical circuit analysis. In Proceedings of the International Symposium on Quality of Electronic Design. 685-692.

B. Swahn and S. Hassoun. 2006. Gate sizing: FinFETs vs. 32nm bulk MOSFETs. In Proceedings of the Design Automation Conference. 528-531.

Synopsys. 2011. Sentaurus TCAD Manual. Retrieved from http://www.synopsys.com.

A. Tang, Y. Yang, C.-Y. Lee, and N. K. Jha. 2015. McPAT-PVT: Delay and power modeling framework for FinFET processor architectures under PVT variations. IEEE Transactions on VLSI Systems 23, 9 (Sep. 2015), 1616-1627.

S. A. Tawfik and V. Kursun. 2007. High speed FinFET domino logic circuits using independent gate-biased double-gate keepers providing dynamically adjusted immunity to noise. In Proceedings of the International Conference on Microelectronics. 175-178.

X. Wang, B. Cheng, A. R. Brown, C. Millar, and A. Asenov. 2014. Accurate simulations of the interplay between process and statistical variability for nanoscale FinFET-based SRAM cell stability. In Proceedings of the 44th European Solid-State Device Research Conference. 349-352.

X. Wang, B. Cheng, A. R. Brown, C. Millar, J. B. Kuang, S. Nassif, and A. Asenov. 2013. Interplay between process-induced and statistical variability in 14-nm CMOS technology double-gate SOI FinFETs. IEEE Transactions on Electronic Devices 60, 8 (Aug. 2013), 2485-2492.

D. Woo et al. 2002. Electrical characteristics of FinFET with vertically nonuniform source/drain doping profile. IEEE Transactions on Nanotechnology 1, 4 (Dec. 2002), 233-237.

C. Wu et al. 2010. High performance 22/20nm FinFET CMOS devices with advanced high-k/metal gate scheme. In Proceedings of the IEEE International Electron Devices Meeting. 27.1.1-27.1.4.

S. Xiong and J. Bokor. 2003. Sensitivity of double-gate and FinFET devices to process variations. IEEE Transactions on Electronic Devices 50 (Nov. 2003), 2255-2261.

T. Yamashita et al. 2011. Sub-25nm FinFET with advanced fin formation and short channel effect engineering. In Proceedings of the IEEE Symposium on VLSI Technology. 14-15.

Received June 2015; revised September 2015; accepted September 2015 